\title{
TITLE:
}

\section{Elliptic Harnack inequalities for symmetric non-local Dirichlet forms}

$\operatorname{AUTHOR}(\mathrm{S})$ :

Chen, Zhen-Qing; Kumagai, Takashi; Wang, Jian

\section{CITATION:}

Chen, Zhen-Qing ... [et al]. Elliptic Harnack inequalities for symmetric non-local Dirichlet forms. Journal de Mathématiques Pures et Appliquées 2019, 125: 1-42

ISSUE DATE:

2019-05

URL:

http://hdl.handle.net/2433/265279

\section{RIGHT:}

(c) 2019. This manuscript version is made available under the Creative Commons Attribution-NonCommercialNoDerivativesl (CC-BY-NC-ND) 4.0 license; The full-text file will be made open to the public on 1 May 2021 in accordance with publisher's 'Terms and Conditions for Self-Archiving'; This is not the published version. Please cite only the published version.この論文は出版社版でありません。引用の際には出版社版をご確認ご利用ください。 


\title{
Elliptic Harnack inequalities for symmetric non-local Dirichlet forms
}

\author{
Zhen-Qing Chen, Takashi Kumagai* and Jian Wang ${ }^{\dagger}$
}

\begin{abstract}
We study relations and characterizations of various elliptic Harnack inequalities for symmetric non-local Dirichlet forms on metric measure spaces. We allow the scaling function be state-dependent and the state space possibly disconnected. Stability of elliptic Harnack inequalities is established under certain regularity conditions and implication for a priori Hölder regularity of harmonic functions is explored. New equivalent statements for parabolic Harnack inequalities of non-local Dirichlet forms are obtained in terms of elliptic Harnack inequalities.
\end{abstract}

\section{$1 \quad$ Introduction and Main Results}

The classical elliptic Harnack inequality asserts that there exists a universal constant $c_{1}=c_{1}(d)$ such that for every $x \in \mathbb{R}^{d}, r>0$ and every non-negative harmonic function $h$ in the ball $B\left(x_{0}, 2 r\right) \subset \mathbb{R}^{d}$,

$$
\operatorname{ess} \sup _{B\left(x_{0}, r\right)} h \leq c \operatorname{ess} \inf _{B\left(x_{0}, r\right)} h .
$$

A celebrated theorem of Moser ( $[\mathrm{M}]$ ) says that such elliptic Harnack inequality holds for nonnegative harmonic functions of any uniformly elliptic divergence operator on $\mathbb{R}^{d}$. One of the important consequences of Moser's elliptic Harnack inequality is that it implies a priori elliptic Hölder regularity (see Definition 1.10 below) for harmonic functions of uniformly elliptic operators of divergence form. Because of the fundamental importance role played by a priori elliptic Hölder regularity for solutions of elliptic and parabolic differential equations, elliptic Harnack inequality and parabolic Harnack inequality, which is a parabolic version of the Harnack inequality (see Definition 1.17below), have been investigated extensively for local operators (diffusions) on various spaces such as manifolds, graphs and metric measure spaces. It is also very important to consider whether such Harnack inequalities are stable under perturbations of the associated quadratic forms and under rough isometries. The stability problem of elliptic Harnack inequality is a difficult one. In [B], R. Bass proved stability of elliptic Harnack inequality under some strong global bounded geometry condition. Quite recently, this assumption has been relaxed significantly by Barlow-Murugan $([\mathrm{BM}])$ to bounded geometry condition.

\footnotetext{
${ }^{*}$ Research partially supported by the Grant-in-Aid for Scientific Research (A) 25247007 and 17H01093, Japan.

${ }^{\dagger}$ Research partially supported by the National Natural Science Foundation of China (No. 11522106), the JSPS postdoctoral fellowship (26.04021), the Fok Ying Tung Education Foundation (No. 151002), National Science Foundation of Fujian Province (No. 2015J01003), the Program for Probability and Statistics: Theory and Application (No. IRTL1704), and Fujian Provincial Key Laboratory of Mathematical Analysis and its Applications (FJKLMAA).
} 
For non-local operators, or equivalently, for discontinuous Markov processes, harmonic functions are required to be non-negative on the whole space in the formulation of Harnack inequalities due to the jumps from the processes; see EHI (elliptic Harnack inequality) in Definition 1.2(i) below. In Bass and Levin [BL, this version of EHI has been established for a class of nonlocal operators. If we only require harmonic functions to be non-negative in the ball $B\left(x_{0}, 2 r\right)$, then the classical elliptic Harnack inequality (1.1) does not need to hold. Indeed, Kassmann [K1] constructed such a counterexample for fractional Laplacian on $\mathbb{R}^{d}$. On the other hand, for nonlocal operators, it is not known whether EHI implies a priori elliptic Hölder regularity (EHR) and we suspect it is not (although parabolic Harnack inequality (PHI) does imply parabolic Hölder regularity and hence EHR, see Theorem 1.19 below). To address this problem, some versions of elliptic Harnack inequalities that imply EHR are considered in some literatures such as [CKP1, CKP2, K1], in connection with the Moser's iteration method. We note that there are now many related work on EHI and EHR for harmonic functions of non-local operators; in addition to the papers mentioned above; see, for instance, [BS, [CK1, CK2, CS, DK, GHH, Ha, HN, K2, Sil] and references therein. This is only a partial list of the vast literature on the subject.

The aim of this paper is to investigate relations among various elliptic Harnack inequalities and to study their stability for symmetric non-local Dirichlet forms under a general setting of metric measure spaces. This paper can be regarded as a continuation of [CKW1, CKW2], which are concerned with the stability of two-sided heat kernel estimates, upper bound heat kernel estimates and PHI for non-local Dirichlet forms on general metric measure spaces. We point out that the setting of this paper is much more general than that of [CKW1, CKW2] in the sense that the scale function in this paper can be state-dependent. As a byproduct, we obtain new equivalent statements for PHI in terms of EHI.

\subsection{Elliptic Harnack inequalities}

Let $(M, d)$ be a locally compact separable metric space, and $\mu$ a positive Radon measure on $M$ with full support. We will refer to such a triple $(M, d, \mu)$ as a metric measure space. Throughout the paper, we assume that $\mu(M)=\infty$. We emphasize that we do not assume $M$ to be connected nor $(M, d)$ to be geodesic.

We consider a regular symmetric Dirichlet form $(\mathcal{E}, \mathcal{F})$ on $L^{2}(M ; \mu)$ of pure jump type; that is,

$$
\mathcal{E}(f, g)=\int_{M \times M \backslash \operatorname{diag}}(f(x)-f(y))(g(x)-g(y)) J(d x, d y), \quad f, g \in \mathcal{F},
$$

where diag denotes the diagonal set $\{(x, x): x \in M\}$ and $J(\cdot, \cdot)$ is a symmetric Radon measure on $M \times M \backslash$ diag. Since $(\mathcal{E}, \mathcal{F})$ is regular, each function $f \in \mathcal{F}$ admits a quasi-continuous version $\tilde{f}$ (see [FOT, Theorem 2.1.3]). In the paper, we will always represent $f \in \mathcal{F}$ by its quasi-continuous version without writing $\tilde{f}$. Let $\mathcal{L}$ be the (negative definite) $L^{2}$-generator of $\mathcal{E}$; this is, $\mathcal{L}$ is the self-adjoint operator in $L^{2}(M ; \mu)$ such that

$$
\mathcal{E}(f, g)=-\langle\mathcal{L} f, g\rangle \text { for all } f \in \mathcal{D}(\mathcal{L}) \text { and } g \in \mathcal{F},
$$

where $\langle\cdot, \cdot\rangle$ denotes the inner product in $L^{2}(M ; \mu)$. Let $\left\{P_{t}\right\}_{t \geq 0}$ be its associated $L^{2}$-semigroup. Associated with the regular Dirichlet form $(\mathcal{E}, \mathcal{F})$ on $L^{2}(M ; \mu)$ is an $\mu$-symmetric Hunt process $X=\left\{X_{t}, t \geq 0 ; \mathbb{P}^{x}, x \in M \backslash \mathcal{N}\right\}$. Here $\mathcal{N}$ is a properly exceptional set for $(\mathcal{E}, \mathcal{F})$ in the sense that $\mu(\mathcal{N})=0$ and $\mathbb{P}^{x}\left(X_{t} \in \mathcal{N}\right.$ for some $\left.t>0\right)=0$ for all $x \in M \backslash \mathcal{N}$. This Hunt process is unique up to a properly exceptional set — see [FOT, Theorem 4.2.8]. We fix $X$ and $\mathcal{N}$, and write $M_{0}=M \backslash \mathcal{N}$. While the semigroup $\left\{P_{t}, t \geq 0\right\}$ associated with $\mathcal{E}$ is defined on $L^{2}(M ; \mu)$, a 
more precise version with better regularity properties can be obtained, if we set, for any bounded Borel measurable function $f$ on $M$,

$$
P_{t} f(x)=\mathbb{E}^{x} f\left(X_{t}\right), \quad x \in M_{0} .
$$

Definition 1.1. Denote by $B(x, r)$ the ball in $(M, d)$ centered at $x$ with radius $r$, and set

$$
V(x, r)=\mu(B(x, r)) .
$$

(i) We say that $(M, d, \mu)$ satisfies the volume doubling property (VD) if there exists a constant $C_{\mu} \geq 1$ such that for all $x \in M$ and $r>0$,

$$
V(x, 2 r) \leq C_{\mu} V(x, r) .
$$

(ii) We say that $(M, d, \mu)$ satisfies the reverse volume doubling property (RVD) if there exist positive constants $d_{1}$ and $c_{\mu}$ such that for all $x \in M$ and $0<r \leq R$,

$$
\frac{V(x, R)}{V(x, r)} \geq c_{\mu}\left(\frac{R}{r}\right)^{d_{1}}
$$

Since $\mu$ has full support on $M$, we have $V(x, r)=\mu(B(x, r))>0$ for every $x \in M$ and $r>0$. The VD condition (1.2) is equivalent to the existence of $d_{2}>0$ and $\widetilde{C}_{\mu} \geq 1$ so that

$$
\frac{V(x, R)}{V(x, r)} \leq \widetilde{C}_{\mu}\left(\frac{R}{r}\right)^{d_{2}} \quad \text { for all } x \in M \text { and } 0<r \leq R .
$$

The RVD condition (1.3) is equivalent to the existence of constants $l_{\mu}>1$ and $\widetilde{c}_{\mu}>1$ so that

$$
V\left(x, l_{\mu} r\right) \geq \widetilde{c}_{\mu} V(x, r) \quad \text { for every } x \in M \text { and } r>0 .
$$

It is known that VD implies RVD if $M$ is connected and unbounded. In fact, it also holds that if $M$ is connected and (1.2) holds for all $x \in M$ and $r \in\left(0, R_{0}\right]$ with some $R_{0}>0$, then (1.3) holds for all $x \in M$ and $0<r \leq R \leq R_{0}$. See, for example [GH1, Proposition 5.1 and Corollary 5.3].

Let $\mathbb{R}_{+}:=[0, \infty)$ and $\phi: M \times \mathbb{R}_{+} \rightarrow \mathbb{R}_{+}$be a strictly increasing continuous function for every fixed $x \in M$ with $\phi(x, 0)=0$ and $\phi(x, 1)=1$ for all $x \in M$, and satisfying that

(i) there exist constants $c_{1}, c_{2}>0$ and $\beta_{2} \geq \beta_{1}>0$ such that

$$
c_{1}\left(\frac{R}{r}\right)^{\beta_{1}} \leq \frac{\phi(x, R)}{\phi(x, r)} \leq c_{2}\left(\frac{R}{r}\right)^{\beta_{2}} \text { for all } x \in M \text { and } 0<r \leq R
$$

(ii) there exists a constant $c_{3} \geq 1$ such that

$$
\phi(y, r) \leq c_{3} \phi(x, r) \text { for all } x, y \in M \text { with } d(x, y) \leq r .
$$

Recall that a set $A \subset M$ is said to be nearly Borel measurable if for any probability measure $\mu_{0}$ on $M$, there are Borel measurable subsets $A_{1}, A_{2}$ of $M$ so that $A_{1} \subset A \subset A_{2}$ and that $\mathbb{P}^{\mu_{0}}\left(X_{t} \in A_{2} \backslash A_{1}\right.$ for some $\left.t \geq 0\right)=0$. The collection of all nearly Borel measurable subsets of $M$ forms a $\sigma$-field, which is called nearly Borel measurable $\sigma$-field. A nearly Borel measurable function $u$ on $M$ is said to be subharmonic (resp. harmonic, superharmonic) in $D$ (with respect 
to the process $X)$ if for any relatively compact subset $U \subset D, t \mapsto u\left(X_{t \wedge \tau_{U}}\right)$ is a uniformly integrable submartingale (resp. martingale, supermartingale) under $\mathbb{P}^{x}$ for $\mathcal{E}$-q.e. $x \in U$. Here $\mathcal{E}$-q.e. stands for $\mathcal{E}$-quasi-everywhere, meaning it holds outside a set having zero 1-capacity with respect to the Dirichlet form $(\mathcal{E}, \mathcal{F})$; see [CF, FOT] for its definition.

For a Borel measurable function $u$ on $M$, we define its non-local tail $\operatorname{Tail}_{\phi}\left(u ; x_{0}, r\right)$ in the ball $B\left(x_{0}, r\right)$ by

$$
\operatorname{Tail}_{\phi}\left(u ; x_{0}, r\right):=\int_{B\left(x_{0}, r\right)^{c}} \frac{|u(z)|}{V\left(x_{0}, d\left(x_{0}, z\right)\right) \phi\left(x_{0}, d\left(x_{0}, z\right)\right)} \mu(d z) .
$$

We need the following definitions for various forms of elliptic Harnack inequalities.

Definition 1.2. (i) We say that elliptic Harnack inequality (EHI) holds for the process $X$, if there exist constants $\delta \in(0,1)$ and $c \geq 1$ such that for every $x_{0} \in M, r>0$ and for every non-negative measurable function $u$ on $M$ that is harmonic in $B\left(x_{0}, r\right)$,

$$
\operatorname{ess}_{\sup _{B\left(x_{0}, \delta r\right)}} u \leq c \operatorname{ess} \inf _{B\left(x_{0}, \delta r\right)} u \text {. }
$$

(ii) We say that non-local elliptic Harnack inequality $\operatorname{EHI}(\phi)$ holds if there exist constants $\delta \in(0,1)$ and $c \geq 1$ such that for every $x_{0} \in M, R>0,0<r \leq \delta R$, and any measurable function $u$ on $M$ that is non-negative and harmonic in $B\left(x_{0}, R\right)$,

$$
\operatorname{ess} \sup _{B\left(x_{0}, r\right)} u \leq c\left(\operatorname{ess~inf}_{B\left(x_{0}, r\right)} u+\phi\left(x_{0}, r\right) \operatorname{Tail}_{\phi}\left(u_{-} ; x_{0}, R\right)\right) .
$$

(iii) We say that non-local weak elliptic Harnack inequality $\operatorname{WEHI}(\phi)$ holds if there exist constants $\varepsilon, \delta \in(0,1)$ and $c \geq 1$ such that for every $x_{0} \in M, R>0,0<r \leq \delta R$, and any measurable function $u$ on $M$ that is non-negative and harmonic in $B\left(x_{0}, R\right)$,

$$
\left(\frac{1}{\mu\left(B\left(x_{0}, r\right)\right)} \int_{B\left(x_{0}, r\right)} u^{\varepsilon} d \mu\right)^{1 / \varepsilon} \leq c\left(\operatorname{ess~inf}_{B\left(x_{0}, r\right)} u+\phi\left(x_{0}, r\right) \operatorname{Tail}_{\phi}\left(u_{-} ; x_{0}, R\right)\right) .
$$

(iv) We say that non-local weak elliptic Harnack inequality $\mathrm{WEHI}^{+}(\phi)$ holds if (iii) holds for any measurable function $u$ on $M$ that is non-negative and superharmonic in $B\left(x_{0}, R\right)$.

Clearly, $\operatorname{EHI}(\phi) \Longrightarrow \mathrm{EHI}+\mathrm{WEHI}(\phi)$, and $\mathrm{WEHI}^{+}(\phi) \Longrightarrow \mathrm{WEHI}(\phi)$. We note that unlike the diffusion case, one needs to assume in the definition of EHI that the harmonic function $u$ is non-negative on the whole space $M$ because the process $X$ can jump all over the places, as mentioned at the beginning of this section.

Remark 1.3. (i) For strongly local Dirichlet forms, $\mathrm{EHI}(\phi)$ is just EHI, and $\mathrm{WEHI}^{+}(\phi)$ (resp. WEHI $(\phi))$ is simply reduced into the following: there exist constants $\varepsilon, \delta \in(0,1)$ and $c \geq 1$ such that for every $x_{0} \in M, 0<r \leq \delta R$, and for every measurable function $u$ that is non-negative and superharmonic (resp. harmonic) in $B\left(x_{0}, R\right)$,

$$
\left(\frac{1}{\mu\left(B\left(x_{0}, r\right)\right)} \int_{B\left(x_{0}, r\right)} u^{\varepsilon} d \mu\right)^{1 / \varepsilon} \leq c \operatorname{essinf}_{B\left(x_{0}, r\right)} u .
$$

The above inequality is called weak Harnack inequality for differential operators. This is why $\operatorname{WEHI}(\phi)$ is called weak Harnack inequality in [CKP1, CKP2, K1]. However for non-local operators this terminology is a bit misleading as it is not implied by EHI. 
(ii) Non-local (weak) elliptic Harnack inequalities have a term involving the non-local tail of harmonic functions, which are essentially due to the jumps of the symmetric Markov processes. This new formulation of Harnack inequalities without requiring the additional positivity on the whole space but adding a non-local tail term first appeared in [K1. The notion of non-local tail of measurable function is formally introduced in [CKP1, CKP2, where non-local (weak) elliptic Harnack inequalities and local behaviors of fractional $p$ Laplacians are investigated. See [DK] and references therein for the background of EHI and WEHI.

To state relations among various notions of elliptic Harnack inequalities and their characterizations, we need a few definitions.

Definition 1.4. (i) We say $\mathrm{J}_{\phi}$ holds if there exists a non-negative symmetric function $J(x, y)$ so that for $\mu \times \mu$-almost all $x, y \in M$,

$$
J(d x, d y)=J(x, y) \mu(d x) \mu(d y),
$$

and

$$
\frac{c_{1}}{V(x, d(x, y)) \phi(x, d(x, y))} \leq J(x, y) \leq \frac{c_{2}}{V(x, d(x, y)) \phi(x, d(x, y))}
$$

We say that $\mathrm{J}_{\phi, \leq}\left(\right.$ resp. $\left.\mathrm{J}_{\phi, \geq}\right)$ holds if (1.7) holds and the upper bound (resp. lower bound) in (1.8) holds for $J(x, y)$.

(ii) We say that $\mathrm{IJ}_{\phi, \leq}$ holds if for $\mu$-almost all $x \in M$ and any $r>0$,

$$
J\left(x, B(x, r)^{c}\right) \leq \frac{c_{3}}{\phi(x, r)} .
$$

Remark 1.5. Under VD and (1.5), $\mathrm{J}_{\phi, \leq}$ implies $\mathrm{IJ}_{\phi, \leq}$, see, e.g., CKW1, Lemma 2.1] or [CK2, Lemma 2.1] for a proof.

For the non-local Dirichlet form $(\mathcal{E}, \mathcal{F})$, we define the carré du-Champ operator $\Gamma(f, g)$ for $f, g \in \mathcal{F}$ by

$$
\Gamma(f, g)(d x)=\int_{y \in M}(f(x)-f(y))(g(x)-g(y)) J(d x, d y) .
$$

Clearly $\mathcal{E}(f, g)=\Gamma(f, g)(M)$. For any $f \in \mathcal{F}_{b}:=\mathcal{F} \cap L^{\infty}(M, \mu), \Gamma(f, f)$ is the unique Borel measure (called the energy measure) on $M$ satisfying

$$
\int_{M} g d \Gamma(f, f)=\mathcal{E}(f, f g)-\frac{1}{2} \mathcal{E}\left(f^{2}, g\right), \quad f, g \in \mathcal{F}_{b}
$$

Let $U \subset V$ be open sets in $M$ with $U \subset \bar{U} \subset V$. We say a non-negative bounded measurable function $\varphi$ is a cutoff function for $U \subset V$, if $\varphi=1$ on $U, \varphi=0$ on $V^{c}$ and $0 \leq \varphi \leq 1$ on $M$.

Definition 1.6. We say that cutoff Sobolev inequality $\operatorname{CSJ}(\phi)$ holds if there exist constants $C_{0} \in(0,1]$ and $C_{1}, C_{2}>0$ such that for every $0<r \leq R$, almost all $x_{0} \in M$ and any $f \in \mathcal{F}$, there exists a cutoff function $\varphi \in \mathcal{F}_{b}$ for $B\left(x_{0}, R\right) \subset B\left(x_{0}, R+r\right)$ so that

$$
\begin{aligned}
\int_{B\left(x_{0}, R+\left(1+C_{0}\right) r\right)} f^{2} d \Gamma(\varphi, \varphi) \leq & C_{1} \int_{U \times U^{*}}(f(x)-f(y))^{2} J(d x, d y) \\
& +\frac{C_{2}}{\phi\left(x_{0}, r\right)} \int_{B\left(x_{0}, R+\left(1+C_{0}\right) r\right)} f^{2} d \mu,
\end{aligned}
$$

where $U=B\left(x_{0}, R+r\right) \backslash B\left(x_{0}, R\right)$ and $U^{*}=B\left(x_{0}, R+\left(1+C_{0}\right) r\right) \backslash B\left(x_{0}, R-C_{0} r\right)$. 
$\operatorname{CSJ}(\phi)$ is introduced in [CKW1, and is used to control the energy of cutoff functions and to characterize the stability of heat kernel estimates for non-local Dirichlet forms. See [CKW1, Remark 1.6] for background on $\operatorname{CSJ}(\phi)$.

Definition 1.7. We say that Poincaré inequality $\mathrm{PI}(\phi)$ holds if there exist constants $C>0$ and $\kappa \geq 1$ such that for any ball $B_{r}=B\left(x_{0}, r\right)$ with $x_{0} \in M$ and $r>0$, and for any $f \in \mathcal{F}_{b}$,

$$
\int_{B_{r}}\left(f-\bar{f}_{B_{r}}\right)^{2} d \mu \leq C \phi\left(x_{0}, r\right) \int_{B_{\kappa r} \times B_{\kappa r}}(f(y)-f(x))^{2} J(d x, d y),
$$

where $\bar{f}_{B_{r}}=\frac{1}{\mu\left(B_{r}\right)} \int_{B_{r}} f d \mu$ is the average value of $f$ on $B_{r}$.

We next introduce the modified Faber-Krahn inequality. For any open set $D \subset M$, let $\mathcal{F}_{D}$ be the $\mathcal{E}_{1}$-closure in $\mathcal{F}$ of $\mathcal{F} \cap C_{c}(D)$, where $\mathcal{E}_{1}(u, u):=\mathcal{E}(u, u)+\int_{M} u^{2} d \mu$. Define

$$
\lambda_{1}(D)=\inf \left\{\mathcal{E}(f, f): f \in \mathcal{F}_{D} \text { with }\|f\|_{2}=1\right\}
$$

the bottom of the Dirichlet spectrum of $-\mathcal{L}$ on $D$.

Definition 1.8. We say that Faber-Krahn inequality $\mathrm{FK}(\phi)$ holds, if there exist positive constants $C$ and $\nu$ such that for any ball $B(x, r)$ and any open set $D \subset B(x, r)$,

$$
\lambda_{1}(D) \geq \frac{C}{\phi(x, r)}(V(x, r) / \mu(D))^{\nu} .
$$

For a set $A \subset M$, define the exit time $\tau_{A}=\inf \left\{t>0: X_{t} \in A^{c}\right\}$.

Definition 1.9. We say that $\mathrm{E}_{\phi}$ holds if there is a constant $c_{1}>1$ such that for all $r>0$ and all $x \in M_{0}$,

$$
c_{1}^{-1} \phi(x, r) \leq \mathbb{E}^{x}\left[\tau_{B(x, r)}\right] \leq c_{1} \phi(x, r) .
$$

We say that $\mathrm{E}_{\phi, \leq}$ (resp. $\mathrm{E}_{\phi, \geq}$ ) holds if the upper bound (resp. lower bound) in the inequality above holds.

Definition 1.10. We say elliptic Hölder regularity (EHR) holds for the process $X$, if there exist constants $c>0, \theta \in(0,1]$ and $\varepsilon \in(0,1)$ such that for every $x_{0} \in M, r>0$ and for every bounded measurable function $u$ on $M$ that is harmonic in $B\left(x_{0}, r\right)$, there is a properly exceptional set $\mathcal{N}_{u} \supset \mathcal{N}$ so that

$$
|u(x)-u(y)| \leq c\left(\frac{d(x, y)}{r}\right)^{\theta}\|u\|_{\infty}
$$

for any $x, y \in B\left(x_{0}, \varepsilon r\right) \backslash \mathcal{N}_{u}$.

Here is the main result of this paper.

Theorem 1.11. Assume that the metric measure space $(M, d, \mu)$ satisfies $\mathrm{VD}$, and $\phi$ satisfies (1.5) and (1.6). Then we have

(i) $\operatorname{WEHI}(\phi) \Longrightarrow \mathrm{EHR}$;

$\mathrm{WEHI}(\phi)+\mathrm{J}_{\phi}+\mathrm{FK}(\phi)+\operatorname{CSJ}(\phi) \Longrightarrow \operatorname{EHI}(\phi)$.

(ii) $\mathrm{J}_{\phi, \leq}+\mathrm{FK}(\phi)+\mathrm{PI}(\phi)+\mathrm{CSJ}(\phi) \Longrightarrow \mathrm{WEHI}^{+}(\phi)$. 
(iii) $\mathrm{EHI}+\mathrm{E}_{\phi, \leq}+\mathrm{J}_{\phi, \leq} \Longrightarrow \mathrm{EHI}(\phi)+\mathrm{FK}(\phi)$;

$\mathrm{EHI}+\mathrm{E}_{\phi}+\mathrm{J}_{\phi, \leq} \Longrightarrow \mathrm{PI}(\phi)$.

As a direct consequence of Theorem 1.11, we have the following statement.

Corollary 1.12. Assume that the metric measure space $(M, d, \mu)$ satisfies $\mathrm{VD}$, and $\phi$ satisfies (1.5) and (1.6). If $\mathrm{J}_{\phi}$ and $\mathrm{E}_{\phi}$ hold, then

$$
\mathrm{FK}(\phi)+\mathrm{PI}(\phi)+\mathrm{CSJ}(\phi) \Longleftrightarrow \mathrm{WEHI}^{+}(\phi) \Longleftrightarrow \mathrm{WEHI}(\phi) \Longleftrightarrow \mathrm{EHI}(\phi) \Longleftrightarrow \mathrm{EHI}
$$

Proof. It follows from Theorem 1.11(ii) that, if $\mathrm{J}_{\phi, \leq}$ holds, then

$$
\mathrm{FK}(\phi)+\mathrm{PI}(\phi)+\mathrm{CSJ}(\phi) \Longrightarrow \mathrm{WEHI}^{+}(\phi) \Longrightarrow \mathrm{WEHI}(\phi) .
$$

By Proposition 4.5(i) below, EHR $+\mathrm{E}_{\phi, \leq} \Longrightarrow \mathrm{FK}(\phi)$. On the other hand, according to Proposition 4.7 below, we have $\mathrm{E}_{\phi}+\mathrm{J}_{\phi, \leq} \Longrightarrow \mathrm{CSJ}(\phi)$. Combining those with Theorem 1.11(i), we can obtain that under $\mathrm{J}_{\phi}$ and $\mathrm{E}_{\phi}$,

$$
\mathrm{WEHI}(\phi) \Longrightarrow \operatorname{EHI}(\phi) \Longrightarrow \mathrm{EHI} .
$$

Furthermore, by Theorem 1.11(iii), if $\mathrm{J}_{\phi, \leq}$ and $\mathrm{E}_{\phi}$ are satisfied, then $\mathrm{EHI} \Longrightarrow \mathrm{FK}(\phi)+\mathrm{PI}(\phi)$. As mentioned above, Proposition 4.7 below shows that $\mathrm{E}_{\phi}+\mathrm{J}_{\phi, \leq} \Longrightarrow \operatorname{CSJ}(\phi)$. Thus, if $\mathrm{J}_{\phi, \leq}$ and $\mathrm{E}_{\phi}$ are satisfied, then

$$
\mathrm{EHI} \Longrightarrow \mathrm{FK}(\phi)+\mathrm{PI}(\phi)+\mathrm{CSJ}(\phi) .
$$

The proof is complete.

\subsection{Stability of elliptic Harnack inequalities}

In this subsection, we study the stability of EHI under some additional assumptions. We mainly follow the framework of $[\mathrm{B}$. For open subsets $A$ and $B$ of $M$ with $A \Subset B$ (that is, $A \subset \bar{A} \subset B$ ), define the relative capacity

$$
\operatorname{Cap}(A, B)=\inf \left\{\mathcal{E}(u, u): u \in \mathcal{F}, u=1 \mathcal{E} \text {-q.e. on } A \text { and } u=0 \mathcal{E} \text {-q.e. on } B^{c}\right\} .
$$

For each $x \in M$ and $r>0$, define

$$
\operatorname{Ext}(x, r)=V(x, r) / \operatorname{Cap}(B(x, r), B(x, 2 r)) .
$$

Our main assumptions are as follows.

Assumption 1.13. (i) $(M, \mu)$ satisfies VD and RVD.

(ii) There is a constant $c_{1}>0$ such that for all $x, y \in M$ with $d(x, y) \leq r$,

$$
\operatorname{Cap}(B(y, r), B(y, 2 r)) \leq c_{1} \operatorname{Cap}(B(x, r), B(x, 2 r)) .
$$

(iii) For any $a \in(0,1]$, there exists a constant $c_{2}:=c_{2, a}>0$ such that for all $x \in M$ and $r>0$,

$$
\operatorname{Cap}(B(x, r), B(x, 2 r)) \leq c_{2} \operatorname{Cap}(B(x, a r), B(x, 2 r)) .
$$


(iv) There exist constants $c_{3}, c_{4}>0$ and $\beta_{2} \geq \beta_{1}>0$ such that for all $x \in M$ and $0<r \leq R$,

$$
c_{3}\left(\frac{R}{r}\right)^{\beta_{1}} \leq \frac{\operatorname{Ext}(x, R)}{\operatorname{Ext}(x, r)} \leq c_{4}\left(\frac{R}{r}\right)^{\beta_{2}} .
$$

Assumption 1.14. For any bounded, non-empty open set $D \subset M$, there exist a properly exceptional set $\mathcal{N}_{D} \supset \mathcal{N}$ and a non-negative measurable function $G_{D}(x, y)$ defined for $x, y \in D \backslash \mathcal{N}_{D}$ such that

(i) $G_{D}(x, y)=G_{D}(y, x)$ for all $(x, y) \in\left(D \backslash \mathcal{N}_{D}\right) \times\left(D \backslash \mathcal{N}_{D}\right) \backslash$ diag.

(ii) for every fixed $y \in D \backslash \mathcal{N}_{D}$, the function $x \mapsto G_{D}(x, y)$ is harmonic in $\left(D \backslash \mathcal{N}_{D}\right) \backslash\{y\}$.

(iii) for every measurable $f \geq 0$ on $D$,

$$
\mathbb{E}^{x}\left[\int_{0}^{\tau_{D}} f\left(X_{t}\right) d t\right]=\int_{D} G_{D}(x, y) f(y) \mu(d y), \quad x \in D \backslash \mathcal{N}_{D} .
$$

The function $G_{D}(x, y)$ satisfying (i)-(iii) of Assumption 1.14 is called the Green function of $X$ in $D$.

Remark 1.15. (i) We will see from Lemmas 5.2 and 5.3 below that, under suitable conditions, the quantity $\operatorname{Ext}(x, r)$ defined above is related to the mean exit time from the ball $B(x, r)$ by the process $X$. Hence, under the conditions, $\operatorname{Ext}(x, r)$ plays the same role of the scaling function $\phi(x, r)$ in the previous subsection.

(ii) From VD and Assumption 1.13 (ii), (iii) and (iv), we can deduce that for every $a \in(0,1]$ and $L>0$, there exists a constant $c_{5}:=c_{a, L, 5} \geq 1$ such that the following holds for all $x, y \in M$ with $d(x, y) \leq r$,

$$
c_{5}^{-1} \operatorname{Cap}(B(y, a L r), B(y, 2 L r)) \leq \operatorname{Cap}(B(x, r), B(x, 2 r)) \leq c_{5} \operatorname{Cap}(B(y, a L r), B(y, 2 L r)) .
$$

(iii) Assumption 1.13 is the same as [BM, Assumption 1.6] except that in their paper the corresponding conditions are assumed to hold for $r \in\left(0, R_{0}\right]$ and for $0<r \leq R \leq R_{0}$ with some $R_{0}>0$. These conditions are called bounded geometry condition in [BM]. However the setting of $[\mathrm{BM}]$ is for strongly local Dirichlet forms with underlying state space $M$ being geodesic. Under these settings and the bounded geometry condition, it is shown in [BM] that there exists an equivalent doubling measure $\widetilde{\mu}$ on $M$ so that Assumption 1.13 holds (i.e., the bounded geometry condition holds globally in large scale as well). Since harmonicity is invariant under time-changes by strictly increasing continuous additive functionals, this enables them to substantially extend the stability result of elliptic Harnack inequality of Bass [B] for diffusions, which was essentially established under the global bounded geometry condition. However the continuity of the processes (i.e. diffusions) and the geodesic property of the underlying state space played a crucial role in [BM]. It is unclear at this stage whether Assumption 1.13 can be replaced by a bounded geometry condition for non-local Dirichlet forms on general metric measure spaces.

The following result gives a stable characterization of EHI.

Theorem 1.16. Under Assumptions 1.13 and 1.14, if $\mathrm{J}_{\text {Ext }}$ holds, then

$\mathrm{FK}(\mathrm{Ext})+\mathrm{PI}(\mathrm{Ext})+\mathrm{CSJ}(\mathrm{Ext}) \Longleftrightarrow \mathrm{WEHI}^{+}(\mathrm{Ext}) \Longleftrightarrow \mathrm{WEHI}(\mathrm{Ext}) \Longleftrightarrow \mathrm{EHI}(\mathrm{Ext}) \Longleftrightarrow \mathrm{EHI}$, where $\mathrm{J}_{\mathrm{Ext}}$ is $\mathrm{J}_{\phi}$ with $\operatorname{Ext}(x, r)$ replacing $\phi(x, r)$, and same for other notions. 


\subsection{Parabolic Harnack inequalities}

As consequences of the main result of this paper, Theorem 1.11 and the stability result of parabolic Harnack inequality in [CKW2, Theorem 1.17], we will present in this subsection new equivalent characterizations of parabolic Hanack inequality in terms of elliptic Harnack inequalities. In this subsection, we always assume that, for each $x \in M$ there is a kernel $J(x, d y)$ so that

$$
J(d x, d y)=J(x, d y) \mu(d y) .
$$

We aim to present some equivalent conditions for parabolic Harnack inequalities in terms of elliptic Harnack inequalities, which can be viewed as a complement to [CKW2. We restrict ourselves to the case that the (scale) function $\phi$ is independent of $x$, i.e. in this subsection, $\phi: \mathbb{R}_{+} \rightarrow \mathbb{R}_{+}$is a strictly increasing continuous function with $\phi(0)=0, \phi(1)=1$ such that there exist constants $c_{3}, c_{4}>0$ and $\beta_{2} \geq \beta_{1}>0$ so that

$$
c_{3}\left(\frac{R}{r}\right)^{\beta_{1}} \leq \frac{\phi(R)}{\phi(r)} \leq c_{4}\left(\frac{R}{r}\right)^{\beta_{2}} \text { for all } 0<r \leq R .
$$

We first give the probabilistic definition of parabolic functions in the general context of metric measure spaces. Let $Z:=\left\{V_{s}, X_{s}\right\}_{s \geq 0}$ be the space-time process corresponding to $X$ where $V_{s}=V_{0}-s$ for all $s \geq 0$. The filtration generated by $Z$ satisfying the usual conditions will be denoted by $\left\{\widetilde{\mathcal{F}}_{s} ; s \geq 0\right\}$. The law of the space-time process $s \mapsto Z_{s}$ starting from $(t, x)$ will be denoted by $\mathbb{P}^{(t, x)}$. For every open subset $D$ of $[0, \infty) \times M$, define $\tau_{D}=\inf \left\{s>0: Z_{s} \notin D\right\}$. We say that a nearly Borel measurable function $u(t, x)$ on $[0, \infty) \times M$ is parabolic (or caloric) in $D=(a, b) \times B\left(x_{0}, r\right)$ for the process $X$ if there is a properly exceptional set $\mathcal{N}_{u}$ of the process $X$ so that for every relatively compact open subset $U$ of $D, u(t, x)=\mathbb{E}^{(t, x)} u\left(Z_{\tau_{U}}\right)$ for every $(t, x) \in U \cap\left([0, \infty) \times\left(M \backslash \mathcal{N}_{u}\right)\right)$.

We next give definitions of parabolic Harnack inequality and Hölder regularity for parabolic functions.

Definition 1.17. (i) We say that parabolic Harnack inequality $\mathrm{PHI}(\phi)$ holds for the process $X$, if there exist constants $0<C_{1}<C_{2}<C_{3}<C_{4}, C_{5}>1$ and $C_{6}>0$ such that for every $x_{0} \in M, t_{0} \geq 0, R>0$ and for every non-negative function $u=u(t, x)$ on $[0, \infty) \times M$ that is parabolic in cylinder $Q\left(t_{0}, x_{0}, C_{4} \phi(R), C_{5} R\right):=\left(t_{0}, t_{0}+C_{4} \phi(R)\right) \times B\left(x_{0}, C_{5} R\right)$,

$$
\operatorname{ess} \sup _{Q_{-}} u \leq C_{6} \operatorname{ess} \inf _{Q_{+}} u,
$$

where $Q_{-}:=\left(t_{0}+C_{1} \phi(R), t_{0}+C_{2} \phi(R)\right) \times B\left(x_{0}, R\right)$ and $Q_{+}:=\left(t_{0}+C_{3} \phi(R), t_{0}+C_{4} \phi(R)\right) \times$ $B\left(x_{0}, R\right)$.

(ii) We say parabolic Hölder regularity $\operatorname{PHR}(\phi)$ holds for the process $X$, if there exist constants $c>0, \theta \in(0,1]$ and $\varepsilon \in(0,1)$ such that for every $x_{0} \in M, t_{0} \geq 0, r>0$ and for every bounded measurable function $u=u(t, x)$ that is parabolic in $Q\left(t_{0}, x_{0}, \phi(r), r\right)$, there is a properly exceptional set $\mathcal{N}_{u} \supset \mathcal{N}$ so that

$$
|u(s, x)-u(t, y)| \leq c\left(\frac{\phi^{-1}(|s-t|)+d(x, y)}{r}\right)^{\theta} \operatorname{ess} \sup _{\left[t_{0}, t_{0}+\phi(r)\right] \times M}|u|
$$

for every $s, t \in\left(t_{0}, t_{0}+\phi(\varepsilon r)\right)$ and $x, y \in B\left(x_{0}, \varepsilon r\right) \backslash \mathcal{N}_{u}$. 
Definition 1.18. We say that UJS holds if there is a symmetric function $J(x, y)$ so that $J(x, d y)=J(x, y) \mu(d y)$, and there is a constant $c>0$ such that for $\mu$-a.e. $x, y \in M$ with $x \neq y$,

$$
J(x, y) \leq \frac{c}{V(x, r)} \int_{B(x, r)} J(z, y) \mu(d z) \quad \text { for every } 0<r \leq \frac{1}{2} d(x, y) .
$$

We define EHR, $\mathrm{E}_{\phi}, \mathrm{E}_{\phi, \leq}, \mathrm{J}_{\phi, \leq}, \mathrm{PI}(\phi)$ and $\operatorname{CSJ}(\phi)$ similarly as in previous subsections but with $\phi(r)$ in place of $\phi(x, r)$. The following stability result of $\operatorname{PHI}(\phi)$ is recently established in CKW2.

Theorem 1.19. ([CKW2, Theorem 1.17]) Suppose that the metric measure space $(M, d, \mu)$ satisfies VD and RVD, and $\phi$ satisfies (1.11). Then the following are equivalent:

(i) $\operatorname{PHI}(\phi)$.

(ii) $\operatorname{PHR}(\phi)+\mathrm{E}_{\phi, \leq}+$ UJS.

(iii) $\mathrm{EHR}+\mathrm{E}_{\phi}+\mathrm{UJS}$.

(iv) $\mathrm{J}_{\phi, \leq}+\mathrm{PI}(\phi)+\mathrm{CSJ}(\phi)+\mathrm{UJS}$.

As a consequence of Theorems 1.11 and 1.19, we have the following statement for the equivalence of $\operatorname{PHI}(\phi)$ in terms of EHI.

Theorem 1.20. Suppose that the metric measure space $(M, d, \mu)$ satisfies VD and RVD, and $\phi$ satisfies (1.11). Then the following are equivalent:

(i) $\operatorname{PHI}(\phi)$.

(ii) $\mathrm{WEHI}^{+}(\phi)+\mathrm{E}_{\phi}+\mathrm{UJS}$.

(iii) $\operatorname{WEHI}(\phi)+\mathrm{E}_{\phi}+\mathrm{UJS}$.

(iv) $\operatorname{EHI}(\phi)+\mathrm{E}_{\phi}+$ UJS.

(v) $\mathrm{EHI}+\mathrm{E}_{\phi}+\mathrm{UJS}+\mathrm{J}_{\phi, \leq}$.

Proof. As indicated in Theorem 1.19, under VD, RVD and (1.11),

$$
\mathrm{PHI}(\phi) \Longleftrightarrow \mathrm{J}_{\phi, \leq}+\mathrm{PI}(\phi)+\operatorname{CSJ}(\phi)+\mathrm{UJS} \Longrightarrow \mathrm{E}_{\phi} .
$$

Then, by Theorem 1.11(ii), (i) $\Longrightarrow$ (ii). (ii) $\Longrightarrow$ (iii) is clear. (iii) $\Longrightarrow$ (i) follows from Theorem 1.11(i) and Theorem 1.19(iii).

Obviously, (i) $\Longrightarrow(\mathrm{v})$ is a consequence of Theorem 1.19 (i), (iii) and (iv). (v) $\Longrightarrow$ (iv) follows from Theorem 1.11 (iii). (iv) $\Longrightarrow$ (iii) is trivial. This completes the proof.

The remainder of this paper is mainly concerned with the proof of Theorem 1.11, the main result of this paper. It is organized as follows. The proofs of Theorem 1.11(i), (ii) and (iii) are given in the next three sections, respectively. In Section 5, we study the relations between the mean of exit time and relative capacity. In particular, the proof of Theorem 1.16 is given there. Finally, a class of symmetric jump processes of variable orders on $\mathbb{R}^{d}$ with state-dependent scaling functions are given in Section [6, for which we apply the main results of this paper to show that all the elliptic Harnack inequalities hold for these processes.

In this paper, we use ":=" as a way of definition. For two functions $f$ and $g$, notation $f \asymp g$ means that there is a constant $c \geq 1$ so that $g / c \leq f \leq c g$. 


\section{Elliptic Harnack inequalities and Hölder regularity}

In this section, we assume that $\mu$ and $\phi$ satisfy VD, (1.5) and (1.6), respectively. We will prove that $\operatorname{WEHI}(\phi)$ implies a priori Hölder regularity for harmonic functions, and study the relation between $\operatorname{WEHI}(\phi)$ and $\operatorname{EHI}(\phi)$.

\section{$2.1 \quad \operatorname{WEHI}(\phi) \Longrightarrow \mathrm{EHR}$}

In this part, we will show that the weak elliptic Harnack inequality implies regularity estimates of harmonic functions in Hölder spaces. We mainly follow the strategy of [DK, Theorem 1.4], part of which is originally due to [M, Sil].

Theorem 2.1. Suppose that $\mathrm{VD}$, (1.5) and $\operatorname{WEHI}(\phi)$ hold. Then there exist constants $\beta \in(0,1)$ and $c>0$ such that for any $x_{0} \in M, r>0$ and harmonic function $u$ on $B\left(x_{0}, r\right)$,

$$
\operatorname{ess}_{B\left(x_{0}, \rho\right)} u \leq c\|u\|_{\infty} \cdot\left(\frac{\rho}{r}\right)^{\beta}, \quad 0<\rho \leq r .
$$

In particular, EHR holds.

Proof. (1) Without loss of generality, we assume the harmonic function $u$ is bounded. Throughout the proof, we fix $x_{0} \in M$, and denote by $B_{r}=B\left(x_{0}, r\right)$ for any $r>0$. For a given bounded harmonic function $u$ on $B_{r}$, we will construct an increasing sequence $\left(m_{n}\right)_{n \geq 1}$ of positive numbers and a decreasing sequence $\left(M_{n}\right)_{n \geq 1}$ that satisfy for any $n \in \mathbb{N} \cup\{0\}$,

$$
\begin{aligned}
& m_{n} \leq u(x) \leq M_{n} \quad \text { for } x \in B_{r \theta^{-n}} \\
& M_{n}-m_{n}=K \theta^{-n \beta} .
\end{aligned}
$$

Here $K=M_{0}-m_{0} \in\left[0,2\|u\|_{\infty}\right]$ with $M_{0}=\|u\|_{\infty}$ and $m_{0}=\operatorname{ess~inf~}_{M} u$, and the constants $\theta=\theta(\delta) \geq \delta^{-1}$ and $\beta=\beta(\delta) \in(0,1)$ are determined later so that

$$
\frac{2-\lambda}{2} \theta^{\beta} \leq 1 \quad \text { for } \lambda:=\left(2^{1+1 / \varepsilon} c\right)^{-1} \in(0,1),
$$

where $\varepsilon, \delta \in(0,1)$ and $c \geq 1$ are the constants in the definition of $\operatorname{WEHI}(\phi)$.

Let us first show that how this construction proves the first desired assertion (2.1). Given $\rho<r$, there is a $j \in \mathbb{N} \cup\{0\}$ such that

$$
r \theta^{-j-1} \leq \rho<r \theta^{-j} .
$$

From (2.2), we conclude

$$
\operatorname{ess~osc}_{B_{\rho}} u \leq \operatorname{ess~osc}_{B_{r \theta^{-j}}} u \leq M_{j}-m_{j}=K \theta^{-j \beta} \leq 2 \theta^{\beta}\|u\|_{\infty}\left(\frac{\rho}{r}\right)^{\beta} .
$$

Set $M_{-n}=M_{0}$ and $m_{-n}=m_{0}$ for any $n \in \mathbb{N}$. Assume that there is a $k \in \mathbb{N}$ and there are $M_{n}$ and $m_{n}$ such that (2.2) holds for $n \leq k-1$. We need to choose $m_{k}, M_{k}$ such that (2.2) still holds for $n=k$. Then the desired assertion follows by induction. For any $x \in M$, set

$$
v(x)=\left(u(x)-\frac{M_{k-1}+m_{k-1}}{2}\right) \frac{2 \theta^{(k-1) \beta}}{K} .
$$


Then the definition of $v$ implies that $|v(x)| \leq 1$ for almost all $x \in B_{r \theta^{-(k-1)}}$. Given $y \in M$ with $d\left(y, x_{0}\right) \geq r \theta^{-(k-1)}$, there is a $j \in \mathbb{N}$ such that

$$
r \theta^{-k+j} \leq d\left(y, x_{0}\right)<r \theta^{-k+j+1} .
$$

For such $y \in M$ and $j \in \mathbb{N}$, on the one hand, we conclude that

$$
\begin{aligned}
\frac{K}{2 \theta^{(k-1) \beta}} v(y) & =u(y)-\frac{M_{k-1}+m_{k-1}}{2} \\
& \leq M_{k-j-1}-m_{k-j-1}+m_{k-j-1}-\frac{M_{k-1}+m_{k-1}}{2} \\
& \leq M_{k-j-1}-m_{k-j-1}-\frac{M_{k-1}-m_{k-1}}{2} \\
& \leq K \theta^{-(k-j-1) \beta}-\frac{K}{2} \theta^{-(k-1) \beta}
\end{aligned}
$$

where in the equalities above we used the fact that if $j>k-1$, then $u(y) \leq M_{0}, m_{k-j-1} \geq m_{0}$ and $M_{0}-m_{0} \leq K \theta^{-(k-j-1) \beta}$. That is,

$$
v(y) \leq 2 \theta^{j \beta}-1 \leq 2\left(\frac{d\left(y, x_{0}\right)}{r \theta^{-k}}\right)^{\beta}-1 .
$$

On the other hand, similarly, we have

$$
\begin{aligned}
\frac{K}{2 \theta^{(k-1) \beta}} v(y) & =u(y)-\frac{M_{k-1}+m_{k-1}}{2} \\
& \geq m_{k-j-1}-M_{k-j-1}+M_{k-j-1}-\frac{M_{k-1}+m_{k-1}}{2} \\
& \geq-\left(M_{k-j-1}-m_{k-j-1}\right)+\frac{M_{k-1}-m_{k-1}}{2} \\
& \geq-K \theta^{-(k-j-1) \beta}+\frac{K}{2} \theta^{-(k-1) \beta}
\end{aligned}
$$

i.e.

$$
v(y) \geq 1-2 \theta^{j \beta} \geq 1-2\left(\frac{d\left(y, x_{0}\right)}{r \theta^{-k}}\right)^{\beta} .
$$

Now, there are two cases:

(i) $\mu\left(\left\{x \in B_{r \theta^{-k}}: v(x) \leq 0\right\}\right) \geq \mu\left(B_{r \theta^{-k}}\right) / 2$.

(ii) $\mu\left(\left\{x \in B_{r \theta^{-k}}: v(x)>0\right\}\right) \geq \mu\left(B_{r \theta^{-k}}\right) / 2$.

In case (i) we aim to show $v(z) \leq 1-\lambda$ for almost every $z \in B_{r \theta^{-k}}$. If this holds true, then for any $z \in B_{r \theta^{-k}}$,

$$
\begin{aligned}
u(z) & \leq \frac{(1-\lambda) K}{2} \theta^{-(k-1) \beta}+\frac{M_{k-1}+m_{k-1}}{2} \\
& =\frac{(1-\lambda) K}{2} \theta^{-(k-1) \beta}+\frac{M_{k-1}-m_{k-1}}{2}+m_{k-1} \\
& =\frac{(1-\lambda) K}{2} \theta^{-(k-1) \beta}+\frac{K}{2} \theta^{-(k-1) \beta}+m_{k-1} \\
& \leq K \theta^{-k \beta}+m_{k-1},
\end{aligned}
$$


where the last inequality follows from the first inequality in (2.3). Thus, we set $m_{k}=m_{k-1}$ and $M_{k}=m_{k}+K \theta^{-k \beta}$, and obtain that $m_{k} \leq u(z) \leq M_{k}$ for almost every $z \in B_{r \theta^{-k}}$.

Consider $w=1-v$ and note that $w \geq 0$ in $B_{r \theta^{-(k-1)}}$. Since in the present setting there is no killing inside $M_{0}$ for the process $X$, constant functions are harmonic, and so $w$ is also harmonic function. Applying $\operatorname{WEHI}(\phi)$ with $w$ on $B_{r \theta^{-(k-1)}}$, we find that

$$
\begin{aligned}
\left(\frac{1}{\mu\left(B_{r \theta^{-k}}\right)}\right. & \left.\int_{B_{r \theta^{-k}}} w^{\varepsilon} d u\right)^{1 / \varepsilon} \\
& \leq c_{1}\left(\operatorname{essinf} B_{r \theta^{-k}} w+\phi\left(x_{0}, r \theta^{-k}\right) \operatorname{Tail}_{\phi}\left(w_{-} ; x_{0}, r \theta^{-(k-1)}\right)\right)
\end{aligned}
$$

Note that, since the constant $c$ in the definition of $\operatorname{WEHI}(\phi)$ may depend on $\delta$ and $\varepsilon$, in the above inequality the constant $c_{1}=c$ could also depend on $\delta$ and $\varepsilon$, thanks to the fact that $\theta^{-1} \leq \delta$. Under case (i)

$$
\left(\frac{1}{\mu\left(B_{r \theta^{-k}}\right)} \int_{B_{r \theta^{-k}}} w^{\varepsilon} d u\right)^{1 / \varepsilon} \geq 2^{-1 / \varepsilon} .
$$

On the other hand, by (2.4), Remark 1.5 and (1.5),

$$
\begin{aligned}
& \phi\left(x_{0}, r \theta^{-(k-1)}\right) \operatorname{Tail}_{\phi}\left(w_{-} ; x_{0}, r \theta^{-(k-1)}\right) \\
& \leq \phi\left(x_{0}, r \theta^{-(k-1)}\right) \int_{B_{r \theta^{-(k-1)}}^{c}} \frac{(1-v(z))_{-}}{V\left(x_{0}, d\left(x_{0}, z\right)\right) \phi\left(x_{0}, d\left(x_{0}, z\right)\right)} \mu(d z) \\
& \leq \phi\left(x_{0}, r \theta^{-(k-1)}\right) \sum_{j=1}^{\infty} \int_{B_{r \theta^{-k+j+1} \backslash B_{r \theta^{-k+j}}}} \frac{(1-v(z))_{-}}{V\left(x_{0}, d\left(x_{0}, z\right)\right) \phi\left(x_{0}, d\left(x_{0}, z\right)\right)} \mu(d z) \\
& \leq \phi\left(x_{0}, r \theta^{-(k-1)}\right) \sum_{j=1}^{\infty} \int_{B_{r \theta^{-k+j+1} \backslash B_{r \theta^{-k+j}}}} \frac{(v(z)-1))_{+}}{V\left(x_{0}, d\left(x_{0}, z\right)\right) \phi\left(x_{0}, d\left(x_{0}, z\right)\right)} \mu(d z) \\
& \leq 2 \phi\left(x_{0}, r \theta^{-(k-1)}\right) \\
& \quad \times \sum_{j=1}^{\infty} \int_{B_{r \theta^{-k+j+1}} \backslash B_{r \theta^{-k+j}}}\left[\left(\frac{d\left(x_{0}, z\right)}{r \theta^{-k}}\right)^{\beta}-1\right] \frac{1}{V\left(x_{0}, d\left(x_{0}, z\right)\right) \phi\left(x_{0}, d\left(x_{0}, z\right)\right)} \mu(d z) \\
& \leq c_{2} \phi\left(x_{0}, r \theta^{-(k-1)}\right) \sum_{j=1}^{\infty} \frac{\theta^{(j+1) \beta}-1}{\phi\left(x_{0}, r \theta^{-k+j}\right)} \\
& \leq c_{3} \sum_{j=1}^{\infty} \theta^{-j \beta_{1}\left(\theta^{j \beta}-1\right),}
\end{aligned}
$$

where $c_{3}>0$ is a constant independent of $k$ and $r$ but depend on $\theta$ and $\beta_{1}$ from (1.5). Hence, by (1.5), (2.5), (2.6) and (2.7), we obtain

$$
\begin{aligned}
{\operatorname{ess~inf~} B_{r \theta^{-k}} w} & \geq\left(c_{1} 2^{1 / \varepsilon}\right)^{-1}-c_{4} \phi\left(x_{0}, r \theta^{-(k-1)}\right) \operatorname{Tail}_{\phi}\left(w_{-} ; x_{0}, r \theta^{-(k-1)}\right) \\
& \geq\left(c 2^{1 / \varepsilon}\right)^{-1}-c_{5} \sum_{j=1}^{\infty} \theta^{-j \beta_{1}}\left(\theta^{j \beta}-1\right) .
\end{aligned}
$$


Note that all the constants $c_{i}(i=1, \ldots, 5)$ may depend on $\theta$. Since for any $\beta \in\left(0, \beta_{1}\right)$,

$$
\sum_{j=1}^{\infty} \theta^{-j \beta_{1}}\left(\theta^{j \beta}-1\right)<\infty
$$

we can choose $l$ large enough (which is independent of $\beta, \theta$ and only depends on $\delta$ ) such that for any $\beta \in\left(0, \beta_{1} / 2\right)$,

$$
\sum_{j=l+1}^{\infty} \theta^{-j \beta_{1}}\left(\theta^{j \beta}-1\right) \leq \sum_{j=l+1}^{\infty} \theta^{-j \beta_{1}}\left(\theta^{j \beta_{1} / 2}-1\right) \leq \sum_{j=l+1}^{\infty} \delta^{j \beta_{1} / 2}<\left(4 c_{5} c 2^{1 / \varepsilon}\right)^{-1} .
$$

Given $l$, one can further take $\beta \in\left(0, \beta_{1} / 2\right)$ small enough such that

$$
\sum_{j=1}^{l} \theta^{-j \beta_{1}}\left(\theta^{j \beta}-1\right) \leq \beta(\log \theta) \sum_{j=1}^{l} \theta^{-j\left(\beta_{1}-\beta\right)} j \leq \beta l \theta^{-\beta_{1} / 2}(\log \theta)<\left(4 c_{5} c 2^{1 / \varepsilon}\right)^{-1} .
$$

(Without loss of generality we may and do assume that $\delta$ in the definition of $\operatorname{WEHI}(\phi)$ is small enough. Thus, the constant $\beta$ here is also independent of $\theta$ and only depends on $\delta$.) Therefore,

$$
\operatorname{ess}_{\inf } B_{r \theta-k} w \geq\left(2 c 2^{1 / \varepsilon}\right)^{-1}=\lambda \text {. }
$$

That is, $v \leq 1-\lambda$ on $B_{r \theta^{-k}}$.

In case (ii), our aim is to show $v \geq-1+\lambda$. This time we set $w=1+v$. Following the arguments above, one sets $M_{k}=M_{k-1}$ and $m_{k}=M_{k}-K \theta^{-k \beta}$ leading to the desired result.

(2) Let $\delta_{0} \in(0,1 / 3)$. Then for almost all $x, y \in B\left(x_{0}, \delta r\right)$, the function $u$ is harmonic on $B\left(x,\left(1-\delta_{0}\right) r\right)$. Note that $d(x, y) \leq 2 \delta_{0} r \leq\left(1-\delta_{0}\right) r$. Applying (2.1), we have

$$
|u(x)-u(y)| \leq \operatorname{ess}_{B(x, d(x, y))} u \leq c\|u\|_{\infty} \cdot\left(\frac{d(x, y)}{\left(1-\delta_{0}\right) r}\right)^{\beta} .
$$

This establishes EHR.

Remark 2.2. The argument above in fact shows that $\mathrm{WEHI}(\phi) \Longrightarrow$ EHR holds for any general jump processes (possibly non-symmetric) that admits no killings inside $M$.

\section{$2.2 \operatorname{WEHI}(\phi)+\mathrm{J}_{\phi}+\mathrm{FK}(\phi)+\operatorname{CSJ}(\phi) \Longrightarrow \operatorname{EHI}(\phi)$}

Let $D$ be an open subset of $M$. Recall that a function $f$ is said to be locally in $\mathcal{F}_{D}$, denoted as $f \in \mathcal{F}_{D}^{\text {loc }}$, if for every relatively compact subset $U$ of $D$, there is a function $g \in \mathcal{F}_{D}$ such that $f=g m$-a.e. on $U$. The following is established in [C].

Lemma 2.3. ([C, Lemma 2.6]) Let $D$ be an open subset of $M$. Suppose $u$ is a function in $\mathcal{F}_{D}^{\text {loc }}$ that is locally bounded on $D$ and satisfies that

$$
\int_{U \times V^{c}}|u(y)| J(d x, d y)<\infty
$$

for any relatively compact open sets $U$ and $V$ of $M$ with $\bar{U} \subset V \subset \bar{V} \subset D$. Then for every $v \in \mathcal{F} \cap C_{c}(D)$, the expression

$$
\int(u(x)-u(y))(v(x)-v(y)) J(d x, d y)
$$

is well defined and finite; it will still be denoted as $\mathcal{E}(u, v)$. 
As noted in $[\mathrm{C},(2.3)]$, since $(\mathcal{E}, \mathcal{F})$ is a regular Dirichlet form on $L^{2}(M ; \mu)$, for any relatively compact open sets $U$ and $V$ with $\bar{U} \subset V$, there is a function $\psi \in \mathcal{F} \cap C_{c}(M)$ such that $\psi=1$ on $U$ and $\psi=0$ on $V^{c}$. Consequently,

$$
\int_{U \times V^{c}} J(d x, d y)=\int_{U \times V^{c}}(\psi(x)-\psi(y))^{2} J(d x, d y) \leq \mathcal{E}(\psi, \psi)<\infty,
$$

so each bounded function $u$ satisfies (2.8).

We say that a nearly Borel measurable function $u$ on $M$ is $\mathcal{E}$-subharmonic (resp. $\mathcal{E}$-harmonic, $\mathcal{E}$-superharmonic) in $D$ if $u \in \mathcal{F}_{D}^{\text {loc }}$ that is locally bounded on $D$, satisfies (2.8) for any relatively compact open sets $U$ and $V$ of $M$ with $\bar{U} \subset V \subset \bar{V} \subset D$, and that

$$
\mathcal{E}(u, \varphi) \leq 0 \quad(\text { resp. }=0, \geq 0) \quad \text { for any } 0 \leq \varphi \in \mathcal{F} \cap C_{c}(D) .
$$

The following is established in [C, Theorem 2.11 and Lemma 2.3] first for harmonic functions, and then extended in [ChK, Theorem 2.9] to subharmonic functions.

Theorem 2.4. Let $D$ be an open subset of $M$, and $u$ be a bounded function. Then $u$ is $\mathcal{E}$ harmonic (resp. $\mathcal{E}$-subharmonic) in $D$ if and only if $u$ is harmonic (resp. subharmonic) in $D$.

The next lemma can be proved by the same argument as that for [CKW1, Proposition 2.3].

Lemma 2.5. Assume that $\mathrm{VD}$, (1.5), (1.6), $\mathrm{J}_{\phi, \leq}$ and $\operatorname{CSJ}(\phi)$ hold. Then there is a constant $c_{0}>0$ such that for every $0<r \leq R$ and almost all $x \in M$,

$$
\operatorname{Cap}(B(x, R), B(x, R+r)) \leq c_{0} \frac{V(x, R+r)}{\phi(x, r)} .
$$

Using this lemma, we can establish the following.

Lemma 2.6. Let $B_{r}=B\left(x_{0}, r\right)$ for some $x_{0} \in M$ and $r>0$. Assume that $u$ is a bounded and $\mathcal{E}$-superharmonic function on $B_{R}$ such that $u \geq 0$ on $B_{R}$. If $\mathrm{VD},(1.5),(1.6), \mathrm{J}_{\phi}, \mathrm{FK}(\phi)$ and $\operatorname{CSJ}(\phi)$ hold, then for any $0<r<R$,

$$
\phi\left(x_{0}, r\right) \operatorname{Tail}_{\phi}\left(u_{+} ; x_{0}, r\right) \leq c\left(\operatorname{ess} \sup _{B_{r}} u+\phi\left(x_{0}, r\right) \operatorname{Tail}_{\phi}\left(u_{-} ; x_{0}, R\right)\right),
$$

where $c>0$ is a constant independent of $u, x_{0}, r$ and $R$.

Proof. According to $\mathrm{J}_{\phi, \leq}, \operatorname{CSJ}(\phi)$ and Lemma 2.5. we can choose $\varphi \in \mathcal{F}_{B_{3 r / 4}}$ related to $\operatorname{Cap}\left(B_{r / 2}, B_{3 r / 4}\right)$ such that

$$
\mathcal{E}(\varphi, \varphi) \leq 2 \operatorname{Cap}\left(B_{r / 2}, B_{3 r / 4}\right) \leq \frac{c_{1} V\left(x_{0}, r\right)}{\phi\left(x_{0}, r\right)} .
$$

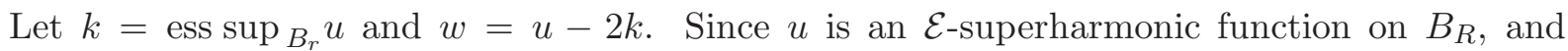
$w \varphi^{2} \in \mathcal{F}_{B_{3 r / 4}}$ with $w<0$ on $B_{r}$,

$$
\begin{aligned}
0 \geq \mathcal{E}\left(u, w \varphi^{2}\right)= & \int_{B_{r} \times B_{r}}(u(x)-u(y))\left(w(x) \varphi^{2}(x)-w(y) \varphi^{2}(y)\right) J(d x, d y) \\
& +2 \int_{B_{r} \times B_{r}^{c}}(u(x)-u(y)) w(x) \varphi^{2}(x) J(d x, d y)
\end{aligned}
$$




$$
=: I_{1}+2 I_{2} \text {. }
$$

For any $x, y \in B_{r}$,

$$
\begin{aligned}
& (u(x)-u(y))\left(w(x) \varphi^{2}(x)-w(y) \varphi^{2}(y)\right) \\
& =(w(x)-w(y))\left(w(x) \varphi^{2}(x)-w(y) \varphi^{2}(y)\right) \\
& =\varphi^{2}(x)(w(x)-w(y))^{2}+w(y)\left(\varphi^{2}(x)-\varphi^{2}(y)\right)(w(x)-w(y)) \\
& \geq \varphi^{2}(x)(w(x)-w(y))^{2}-\frac{1}{8}(\varphi(x)+\varphi(y))^{2}(w(x)-w(y))^{2}-2 w^{2}(y)(\varphi(x)-\varphi(y))^{2},
\end{aligned}
$$

where we used the fact that $a b \geq-\left(\frac{1}{8} a^{2}+2 b^{2}\right)$ for all $a, b \in \mathbb{R}$ in the inequality above. Hence,

$$
\begin{aligned}
I_{1} \geq & \int_{B_{r} \times B_{r}} \varphi^{2}(x)(w(x)-w(y))^{2} J(d x, d y) \\
& -\frac{1}{8} \int_{B_{r} \times B_{r}}(\varphi(x)+\varphi(y))^{2}(w(x)-w(y))^{2} J(d x, d y) \\
& -2 \int_{B_{r} \times B_{r}} w^{2}(y)(\varphi(x)-\varphi(y))^{2} J(d x, d y) \\
\geq & \frac{1}{2} \int_{B_{r} \times B_{r}} \varphi^{2}(x)(w(x)-w(y))^{2} J(d x, d y) \\
& -8 k^{2} \int_{B_{r} \times B_{r}}(\varphi(x)-\varphi(y))^{2} J(d x, d y) \\
\geq & -8 k^{2} \int_{B_{r} \times B_{r}}(\varphi(x)-\varphi(y))^{2} J(d x, d y),
\end{aligned}
$$

where in the second inequality we have used the symmetry property of $J(d x, d y)$ and the fact that $w^{2} \leq 4 k^{2}$ on $B_{r}$.

On the other hand, by the definition of $w$, it is easy to see that for any $x \in B_{r}$ and $y \notin B_{r}$

$$
\begin{aligned}
(u(x)-u(y)) w(x) & \geq k(u(y)-k)_{+}-2 k \mathbf{1}_{\{u(y) \leq k\}}(u(x)-u(y))_{+} \\
& \geq k(u(y)-k)_{+}-2 k(u(x)-u(y))_{+},
\end{aligned}
$$

and so

$$
\begin{aligned}
I_{2} \geq & \int_{B_{r} \times B_{r}^{c}} k(u(y)-k)_{+} \varphi^{2}(x) J(d x, d y) \\
& -\int_{B_{r} \times B_{r}^{c}} 2 k(u(x)-u(y))_{+} \varphi^{2}(x) J(d x, d y) \\
= & : I_{21}-I_{22} .
\end{aligned}
$$

Furthermore, since $(u(y)-k)_{+} \geq u_{+}(y)-k$, we find that

$$
\begin{aligned}
I_{21} & \geq k \int_{B_{r} \times B_{r}^{c}} u_{+}(y) \varphi^{2}(x) J(d x, d y)-k^{2} \int_{B_{r} \times B_{r}^{c}} \varphi^{2}(x) J(d x, d y) \\
& \geq k \mu\left(B_{r / 2}\right) \inf _{x \in B_{r / 2}} \int_{B_{r}^{c}} u_{+}(y) J(x, d y)-k^{2} \int_{B_{r} \times B_{r}^{c}} \varphi^{2}(x) J(d x, d y) \\
& \geq c_{1} k V\left(x_{0}, r\right) \operatorname{Tail}_{\phi}\left(u_{+} ; x_{0}, r\right)-k^{2} \int_{B_{r} \times B_{r}^{c}} \varphi^{2}(x) J(d x, d y),
\end{aligned}
$$


where in the second inequality we have used the fact that $\varphi=1$ on $B_{r / 2}$, and in the last inequality we have used $\mathrm{J}_{\phi, \geq}$ and the fact that for all $x \in B_{r / 2}$ and $z \in B_{r}^{c}$,

$$
\frac{V(x, d(x, z))}{V\left(x_{0}, d\left(x_{0}, z\right)\right)} \frac{\phi(x, d(x, z))}{\phi\left(x_{0}, d\left(x_{0}, z\right)\right)} \leq c^{\prime}\left(1+\frac{d\left(x, x_{0}\right)}{d\left(x_{0}, z\right)}\right)^{d_{2}+\beta_{2}} \leq c^{\prime \prime}
$$

thanks to VD, (1.5) and (1.6). Also, since $u \geq 0$ on $B_{R}$, we can check that

$$
\begin{aligned}
I_{22} & \leq 2 k \int_{B_{r} \times\left(B_{R} \backslash B_{r}\right)} k \varphi^{2}(x) J(d x, d y)+2 k \int_{B_{r} \times B_{R}^{c}}\left(k+u_{-}(y)\right) \varphi^{2}(x) J(d x, d y) \\
& \leq 2 k^{2} \int_{B_{r} \times B_{r}^{c}} \varphi^{2}(x) J(d x, d y)+c_{2} k^{2} \frac{V\left(x_{0}, r\right)}{\phi\left(x_{0}, r\right)}+c_{2} k V\left(x_{0}, r\right) \operatorname{Tail}_{\phi}\left(u_{-} ; x_{0}, R\right),
\end{aligned}
$$

where the second term of the last inequality follows from Remark 1.5] and (1.6), and in the third term we have used $\mathrm{J}_{\phi, \leq}$.

By the estimates for $I_{21}$ and $I_{22}$, we get that

$$
\begin{aligned}
I_{2} \geq & -3 k^{2} \int_{B_{r} \times B_{r}^{c}} \varphi^{2}(x) J(d x, d y)+c_{1} k V\left(x_{0}, r\right) \operatorname{Tail}_{\phi}\left(u_{+} ; x_{0}, r\right) \\
& -c_{2} k^{2} \frac{V\left(x_{0}, r\right)}{\phi\left(x_{0}, r\right)}-c_{2} k V\left(x_{0}, r\right) \operatorname{Tail}_{\phi}\left(u_{-} ; x_{0}, R\right) .
\end{aligned}
$$

This along with the estimate for $I_{1}$ yields that

$$
V\left(x_{0}, r\right) \operatorname{Tail}_{\phi}\left(u_{+} ; x_{0}, r\right) \leq c_{3}\left[k\left(\frac{V\left(x_{0}, r\right)}{\phi\left(x_{0}, r\right)}+\mathcal{E}(\varphi, \varphi)\right)+V\left(x_{0}, r\right) \operatorname{Tail}_{\phi}\left(u_{-} ; x_{0}, R\right)\right] .
$$

Then, combining this inequality with (2.9) proves the desired assertion.

We also need the following result. Since the proof is essentially the same as that of CKW1, Proposition 4.10], we omit it here.

Proposition 2.7. Let $x_{0} \in M$ and $R>0$. Assume VD, (1.5), (1.6), $\mathrm{J}_{\phi, \leq}, \mathrm{FK}(\phi)$ and $\operatorname{CSJ}(\phi)$ hold, and let $u$ be a bounded $\mathcal{E}$-subharmonic in $B\left(x_{0}, R\right)$. Then for any $\delta>0$,

$$
\operatorname{ess~sup}_{B\left(x_{0}, R / 2\right)} u \leq c_{1}\left[\left(\frac{\left(1+\delta^{-1}\right)^{1 / \nu}}{V\left(x_{0}, R\right)} \int_{B\left(x_{0}, R\right)} u^{2} d \mu\right)^{1 / 2}+\delta \phi\left(x_{0}, R\right) \operatorname{Tail}_{\phi}\left(u ; x_{0}, R / 2\right)\right],
$$

where $\nu$ is the constant in $\mathrm{FK}(\phi)$, and $c_{1}>0$ is a constant independent of $x_{0}, R, \delta$ and $u$.

We are in a position to present the main statement in this subsection.

Theorem 2.8. Let $B_{r}\left(x_{0}\right)=B\left(x_{0}, r\right)$ for some $x_{0} \in M$ and $r>0$. Assume that $u$ is a bounded and $\mathcal{E}$-harmonic function on $B_{R}\left(x_{0}\right)$ such that $u \geq 0$ on $B_{R}\left(x_{0}\right)$. Assume that VD, (1.5), (1.6) $, \mathrm{J}_{\phi}, \mathrm{FK}(\phi)$ and $\operatorname{CSJ}(\phi)$, and $\operatorname{WEHI}(\phi)$ hold. Then the following estimate holds for any $0<r<\delta_{0} R$,

$$
\operatorname{ess} \sup _{B_{r / 2}\left(x_{0}\right)} u \leq c\left(\operatorname{ess}_{\inf } B_{r}\left(x_{0}\right) u+\phi\left(x_{0}, r\right) \operatorname{Tail}_{\phi}\left(u_{-} ; x_{0}, R\right)\right),
$$

where $\delta_{0} \in(0,1)$ is the constant $\delta$ in $\operatorname{WEHI}(\phi)$ and $c>0$ is a constant independent of $x_{0}, r, R$ and $u$. This is,

$$
\mathrm{WEHI}(\phi)+\mathrm{J}_{\phi}+\mathrm{FK}(\phi)+\mathrm{CSJ}(\phi) \Longrightarrow \operatorname{EHI}(\phi)
$$


Proof. Note that $u_{+}$is a bounded and $\mathcal{E}$-subharmonic function on $B_{R}\left(x_{0}\right)$. According to Proposition 2.7. for any $0<\delta<1$ and $0<\rho<R$,

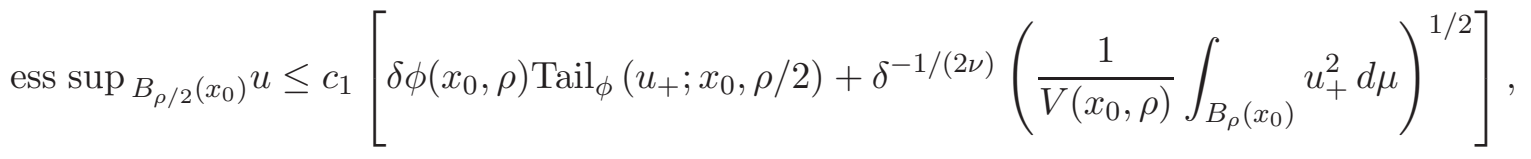

where $c_{1}>0$ is a constant independent of $x_{0}, \rho, u$ and $\delta$. The inequality above along with Lemma 2.6 yields that

$$
\begin{aligned}
\operatorname{ess}_{\sup _{B_{\rho / 2}\left(x_{0}\right)} u \leq c_{2}(} & \delta^{-1 /(2 \nu)}\left(\frac{1}{V\left(x_{0}, \rho\right)} \int_{B_{\rho}\left(x_{0}\right)} u_{+}^{2} d \mu\right)^{1 / 2} \\
& \left.+\delta \operatorname{ess} \sup _{B_{\rho}\left(x_{0}\right)} u+\delta \phi\left(x_{0}, \rho\right) \operatorname{Tail}_{\phi}\left(u_{-} ; x_{0}, R\right)\right)
\end{aligned}
$$

For any $1 / 2 \leq \sigma^{\prime} \leq \sigma \leq 1$ and $z \in B_{\sigma^{\prime} r}\left(x_{0}\right)$, applying the inequality above with $B_{\rho}\left(x_{0}\right)=$ $B_{\left(\sigma-\sigma^{\prime}\right) r}(z)$, we get that there is a constant $c_{3}>1$ such that

$$
\begin{aligned}
u(z) \leq c_{3}( & \frac{\delta^{-1 /(2 \nu)}}{\left(\sigma-\sigma^{\prime}\right)^{d_{2} / 2}}\left(\frac{1}{V\left(x_{0}, \sigma r\right)} \int_{B_{\sigma r}\left(x_{0}\right)} u^{2} d \mu\right)^{1 / 2} \\
& \left.+\delta \operatorname{ess} \sup _{B_{\sigma r}\left(x_{0}\right)} u+\delta \phi\left(x_{0}, r\right) \operatorname{Tail}_{\phi}\left(u_{-} ; x_{0}, R\right)\right)
\end{aligned}
$$

where we have used the facts that $B_{\left(\sigma-\sigma^{\prime}\right) r}(z) \subset B_{\sigma r}\left(x_{0}\right)$ for any $z \in B_{\sigma^{\prime} r}\left(x_{0}\right)$, and

$$
\frac{V\left(x_{0}, \sigma r\right)}{V\left(z,\left(\sigma-\sigma^{\prime}\right) r\right)} \leq c^{\prime}\left(1+\frac{d\left(x_{0}, z\right)+\sigma r}{\left(\sigma-\sigma^{\prime}\right) r}\right)^{d_{2}} \leq c^{\prime \prime}\left(1+\frac{\sigma r+\sigma^{\prime} r}{\left(\sigma-\sigma^{\prime}\right) r}\right)^{d_{2}} \leq \frac{c^{\prime \prime \prime}}{\left(\sigma-\sigma^{\prime}\right)^{d_{2}}}
$$

thanks to VD. Therefore,

$$
\begin{aligned}
\operatorname{ess} \sup _{B_{\sigma^{\prime}}\left(x_{0}\right)} u \leq c_{3}( & \frac{\delta^{-1 /(2 \nu)}}{\left(\sigma-\sigma^{\prime}\right)^{d_{2} / 2}}\left(\frac{1}{V\left(x_{0}, \sigma r\right)} \int_{B_{\sigma r}\left(x_{0}\right)} u^{2} d \mu\right)^{1 / 2} \\
& \left.+\delta \operatorname{ess} \sup _{B_{\sigma r}\left(x_{0}\right)} u+\delta \phi\left(x_{0}, r\right) \operatorname{Tail}_{\phi}\left(u_{-} ; x_{0}, R\right)\right) .
\end{aligned}
$$

In particular, choosing $\delta=\frac{1}{4 c_{3}}$ in the inequality above, we arrive at

$\operatorname{ess} \sup _{B_{\sigma^{\prime} r}\left(x_{0}\right)} u \leq \frac{1}{4} \operatorname{ess} \sup _{B_{\sigma r}\left(x_{0}\right)} u$

$$
+\frac{c_{4}}{\left(\sigma-\sigma^{\prime}\right)^{d_{2} / 2}}\left(\frac{1}{V\left(x_{0}, \sigma r\right)} \int_{B_{\sigma r}\left(x_{0}\right)} u^{2} d \mu\right)^{1 / 2}+c_{4} \phi\left(x_{0}, r\right) \operatorname{Tail}_{\phi}\left(u_{-} ; x_{0}, R\right) .
$$

Since

$$
\frac{c_{4}}{\left(\sigma-\sigma^{\prime}\right)^{d_{2} / 2}}\left(\frac{1}{V\left(x_{0}, \sigma r\right)} \int_{B_{\sigma r}\left(x_{0}\right)} u^{2} d \mu\right)^{1 / 2}
$$




$$
\begin{aligned}
& \leq \frac{c_{4}}{\left(\sigma-\sigma^{\prime}\right)^{d_{2} / 2}} \frac{\left(\operatorname{ess} \sup _{B_{\sigma r}\left(x_{0}\right)} u\right)^{(2-q) / 2}}{V\left(x_{0}, \sigma r\right)^{1 / 2}}\left(\int_{B_{\sigma r}\left(x_{0}\right)}|u|^{q} d \mu\right)^{1 / 2} \\
& \leq \frac{1}{4} \operatorname{ess} \sup _{B_{\sigma r}\left(x_{0}\right)} u+\frac{c_{4}^{\prime}}{\left(\sigma-\sigma^{\prime}\right)^{d_{2} / q}}\left(\frac{1}{V\left(x_{0}, \sigma r\right)} \int_{B_{\sigma r}\left(x_{0}\right)}|u|^{q} d \mu\right)^{1 / q},
\end{aligned}
$$

where in the last inequality we applied the standard Young inequality with exponent $2 / q$ and $2 /(2-q)$ with any $0<q<2$, we have for any $0<q<2$ and $1 / 2 \leq \sigma^{\prime} \leq \sigma \leq 1$,

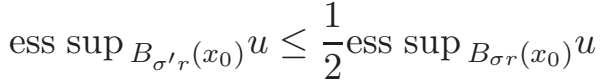

$$
\begin{aligned}
& +\frac{c_{5}}{\left(\sigma-\sigma^{\prime}\right)^{d_{2} / q}}\left[\left(\frac{1}{V\left(x_{0}, \sigma r\right)} \int_{B_{\sigma r}\left(x_{0}\right)} u^{q} d \mu\right)^{1 / q}+\phi\left(x_{0}, r\right) \operatorname{Tail}_{\phi}\left(u_{-} ; x_{0}, R\right)\right] \\
& \leq \frac{1}{2} \operatorname{ess} \sup _{B_{\sigma r}\left(x_{0}\right)} u \\
& +\frac{c_{5}^{\prime}}{\left(\sigma-\sigma^{\prime}\right)^{d_{2} / q}}\left[\left(\frac{1}{V\left(x_{0}, r\right)} \int_{B_{r}\left(x_{0}\right)} u^{q} d \mu\right)^{1 / q}+\phi\left(x_{0}, r\right) \operatorname{Tail}_{\phi}\left(u_{-} ; x_{0}, R\right)\right] .
\end{aligned}
$$

According to Lemma 2.9 below, we find that

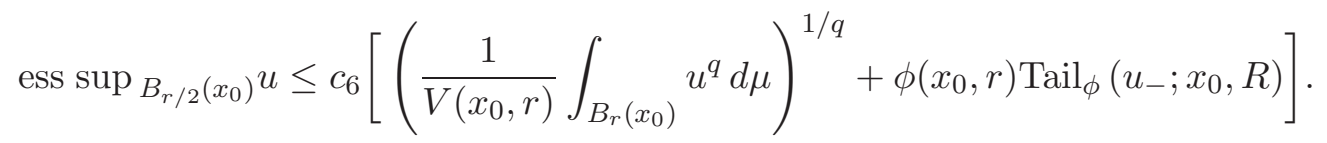

To conclude the proof, we combine the above inequality with $\operatorname{WEHI}(\phi)$ and Theorem 2.4. by setting $q=\varepsilon$.

The following lemma is taken from [GG, Lemma 1.1], which has been used in the proof above.

Lemma 2.9. Let $f(t)$ be a non-negative bounded function defined for $0 \leq T_{0} \leq t \leq T_{1}$. Suppose that for $T_{0} \leq t \leq s \leq T_{1}$ we have

$$
f(t) \leq A(s-t)^{-\alpha}+B+\theta f(s)
$$

where $A, B, \alpha, \theta$ are non-negative constants, and $\theta<1$. Then there exists a positive constant $c$ depending only on $\alpha$ and $\theta$ such that for every $T_{0} \leq r \leq R \leq T_{1}$, we have

$$
f(r) \leq c\left(A(R-r)^{-\alpha}+B\right) .
$$

\section{$3 \quad$ Sufficient condition for $\mathrm{WEHI}^{+}(\phi)$}

In this section, we will establish the following, which gives a sufficient condition for $\mathrm{WEHI}^{+}(\phi)$.

Theorem 3.1. Assume that VD, (1.5), (1.6), $\mathrm{J}_{\phi, \leq}, \mathrm{FK}(\phi), \mathrm{PI}(\phi)$ and $\mathrm{CSJ}(\phi)$ hold. Then, $\mathrm{WEHI}^{+}(\phi)$ holds. More precisely, there exist constants $\varepsilon \in(0,1)$ and $c \geq 1$ such that for all $x_{0} \in M, 0<r<R /(60 \kappa)$ and any bounded $\mathcal{E}$-superharmonic function $u$ on $B_{R}:=B\left(x_{0}, R\right)$ with $u \geq 0$ on $B_{R}$,

$$
\left(\frac{1}{\mu\left(B_{r}\right)} \int_{B_{r}} u^{\varepsilon} d \mu\right)^{1 / \varepsilon} \leq c\left(\operatorname{ess~inf}_{B_{r}} u+\phi\left(x_{0}, r\right) \operatorname{Tail}_{\phi}\left(u_{-} ; x_{0}, R\right)\right),
$$

where $\kappa \geq 1$ is the constant in $\mathrm{PI}(\phi)$ and $B_{r}=B\left(x_{0}, r\right)$. 
Throughout this section, we always assume that $\mu$ and $\phi$ satisfy VD, (1.5) and (1.6), respectively. To prove Theorem 3.1 we mainly follow [CKP2], which is originally due to [DT]. Since we essentially make use of $\operatorname{CSJ}(\phi)$, some nontrivial modifications are required. We begin with the following result, which easily follows from [CKW2, Corollary 4.12].

Lemma 3.2. Let $B_{r}=B\left(x_{0}, r\right)$ for some $x_{0} \in M$ and $r>0$. Assume that $u$ is a bounded and $\mathcal{E}$-superharmonic function on $B_{R}$ such that $u \geq 0$ on $B_{R}$. For any $a, l>0$ and $b>1$, define

$$
v=\left[\log \left(\frac{a+l}{u+l}\right)\right]_{+} \wedge \log b .
$$

If $\mathrm{VD}$, (1.5), (1.6), $\mathrm{J}_{\phi, \leq}, \mathrm{PI}(\phi)$ and $\mathrm{CSJ}(\phi)$ hold, then for any $l>0$ and $0<r \leq R /(2 \kappa)$,

$$
\frac{1}{V\left(x_{0}, r\right)} \int_{B_{r}}\left(v-\bar{v}_{B_{r}}\right)^{2} d \mu \leq c_{1}\left(1+\frac{\phi\left(x_{0}, r\right) \operatorname{Tail}_{\phi}\left(u_{-} ; x_{0}, R\right)}{l}\right)
$$

where $\kappa \geq 1$ is the constant in $\mathrm{PI}(\phi), \bar{v}_{B_{r}}=\frac{1}{\mu\left(B_{r}\right)} \int_{B_{r}} v d \mu$ and $c_{1}$ is a constant independent of $u, x_{0}, r, R$ and $l$.

Lemma 3.3. Let $B_{r}=B\left(x_{0}, r\right)$ for some $x_{0} \in M$ and $r>0$. Assume that $u$ is a bounded and $\mathcal{E}$-superharmonic function on $B_{R}$ such that $u \geq 0$ on $B_{R}$. Assume that $\mathrm{VD}$, (1.5), (1.6), $\mathrm{J}_{\phi, \leq}$, $\mathrm{FK}(\phi), \operatorname{PI}(\phi)$ and $\operatorname{CSJ}(\phi)$ hold. Suppose that there exist constants $\lambda>0$ and $\sigma \in(0,1]$ such that

$$
\mu\left(B_{r} \cap\{u \geq \lambda\}\right) \geq \sigma \mu\left(B_{r}\right)
$$

for some $r$ with $0<r<R /(12 \kappa)$, where $\kappa \geq 1$ is the constant in $\operatorname{PI}(\phi)$. Then there exists a constant $c_{1}>0$ such that

$$
\frac{\mu\left(B_{6 r} \cap\left\{u \leq 2 \delta \lambda-\frac{1}{2} \phi\left(x_{0}, r\right) \operatorname{Tail}_{\phi}\left(u_{-} ; x_{0}, R\right)\right\}\right)}{\mu\left(B_{6 r}\right)} \leq \frac{c_{1}}{\sigma \log \frac{1}{2 \delta}}
$$

holds for all $\delta \in(0,1 / 4)$, where $c_{1}$ is a constant independent of $u, x_{0}, r, R, \sigma, \lambda$ and $\delta$.

Proof. Taking $l=\frac{1}{2} \phi\left(x_{0}, r\right) \operatorname{Tail}_{\phi}\left(u_{-} ; x_{0}, R\right), a=\lambda$ with $\lambda>0$, and $b=\frac{1}{2 \delta}$ with $\delta \in(0,1 / 4)$ in Lemma 3.2, we get that for all $\lambda>0$ and $0<r<R /(12 \kappa)$,

$$
\frac{1}{V\left(x_{0}, 6 r\right)} \int_{B_{6 r}}\left|v-\bar{v}_{B_{6 r}}\right| d \mu \leq\left(\frac{1}{V\left(x_{0}, 6 r\right)} \int_{B_{6 r}}\left(v-\bar{v}_{B_{6 r}}\right)^{2} d \mu\right)^{1 / 2} \leq c_{1},
$$

where

$$
v=\min \left\{\left[\log \left(\frac{\lambda+l}{u+l}\right)\right]_{+}, \log \frac{1}{2 \delta}\right\} .
$$

Notice that by the definition of $v$, we have $\{v=0\}=\{u \geq \lambda\}$. Hence, by (3.1) and VD, for some $r$ with $0<r<R /(12 \kappa)$,

$$
\mu\left(B_{6 r} \cap\{v=0\}\right) \geq c^{\prime} \sigma \mu\left(B_{6 r}\right)
$$

and so

$$
\log \frac{1}{2 \delta}=\frac{1}{\mu\left(B_{6 r} \cap\{v=0\}\right)} \int_{B_{6 r} \cap\{v=0\}}\left(\log \frac{1}{2 \delta}-v\right) d \mu
$$




$$
\begin{aligned}
& \leq \frac{1}{c^{\prime} \sigma} \frac{1}{\mu\left(B_{6 r}\right)} \int_{B_{6 r}}\left(\log \frac{1}{2 \delta}-v\right) d \mu \\
& \leq \frac{1}{c^{\prime} \sigma}\left(\log \frac{1}{2 \delta}-\bar{v}_{B_{6 r}}\right) .
\end{aligned}
$$

Thus, integrating the previous inequality over $B_{6 r} \cap\left\{v=\log \frac{1}{2 \delta}\right\}$, we obtain

$$
\begin{aligned}
\left(\log \frac{1}{2 \delta}\right) \mu\left(B_{6 r} \cap\left\{v=\log \frac{1}{2 \delta}\right\}\right) & \leq \frac{1}{c^{\prime} \sigma} \int_{B_{6 r} \cap\left\{v=\log \frac{1}{2 \delta}\right\}}\left(\log \frac{1}{2 \sigma}-\bar{v}_{B_{6 r}}\right) d \mu \\
& \leq \frac{1}{c^{\prime} \sigma} \int_{B_{6 r}}\left|v-\bar{v}_{B_{6 r}}\right| d \mu \\
& \leq \frac{c_{2}}{\sigma} V\left(x_{0}, 6 r\right),
\end{aligned}
$$

where in the last inequality we have used (3.2). Therefore, for all $0<\delta<1 / 4$,

$$
\mu\left(B_{6 r} \cap\{u+l \leq 2 \delta(\lambda+l)\}\right) \leq \frac{c_{2}}{\sigma \log \frac{1}{2 \delta}} V\left(x_{0}, 6 r\right),
$$

which proves the desired assertion.

For any $x \in M$ and $r>0$, set $B_{r}(x)=B(x, r)$. For a ball $B \subset M$ and a function $w$ on $B$, write

$$
I(w, B)=\int_{B} w^{2} d \mu .
$$

The following lemma can be proved similarly as the of [CKW1, Lemma 4.8].

Lemma 3.4. Suppose VD, (1.5), (1.6), $\mathrm{J}_{\phi, \leq}, \mathrm{FK}(\phi)$ and $\mathrm{CSJ}(\phi)$ hold. For $x_{0} \in M, R, r_{1}, r_{2}>$ 0 with $r_{1} \in\left[\frac{1}{2} R, R\right]$ and $r_{1}+r_{2} \leq R$, let $u$ be an $\mathcal{E}$-subharmonic function on $B_{R}\left(x_{0}\right)$, and $v=(u-\theta)_{+}$for some $\theta>0$. Set $I_{0}=I\left(u, B_{r_{1}+r_{2}}\left(x_{0}\right)\right)$ and $I_{1}=I\left(v, B_{r_{1}}\left(x_{0}\right)\right)$. We have

$$
I_{1} \leq \frac{c_{1}}{\theta^{2 \nu} V\left(x_{0}, R\right)^{\nu}} I_{0}^{1+\nu}\left(1+\frac{r_{1}}{r_{2}}\right)^{\beta_{2}}\left[1+\left(1+\frac{r_{1}}{r_{2}}\right)^{d_{2}+\beta_{2}-\beta_{1}} \frac{\phi\left(x_{0}, R\right) \operatorname{Tail}_{\phi}\left(u ; x_{0}, R / 2\right)}{\theta}\right]
$$

where $\nu$ is the constant in $\mathrm{FK}(\phi), d_{2}$ is the constant in (1.4), $\beta_{1}$ and $\beta_{2}$ are the constants in (1.5), and $c_{1}$ is a constant independent of $\theta, x_{0}, R, r_{1}, r_{2}$ and $u$.

We also need the following elementary iteration lemma, see, e.g., [Giu, Lemma 7.1] or [CKW1, Lemma 4.9].

Lemma 3.5. Let $\beta>0$ and let $\left\{A_{j}\right\}$ be a sequence of real positive numbers such that

$$
A_{j+1} \leq c_{0} b^{j} A_{j}^{1+\beta}, \quad j \geq 1
$$

with $c_{0}>0$ and $b>1$. If

$$
A_{0} \leq c_{0}^{-1 / \beta} b^{-1 / \beta^{2}}
$$

then we have

$$
A_{j} \leq b^{-j / \beta} A_{0}, \quad j \geq 0,
$$

which in particular yields $\lim _{j \rightarrow \infty} A_{j}=0$. 
The following proposition gives us the infimum of the superharmonic function. This extends the analogous expansion of positivity in the local setting, which is a key step towards $\mathrm{WEHI}^{+}(\phi)$.

Proposition 3.6. Let $B_{r}=B\left(x_{0}, r\right)$ for some $x_{0} \in M$ and any $r>0$. Assume that $u$ is a bounded and $\mathcal{E}$-superharmonic function on $B_{R}$ such that $u \geq 0$ on $B_{R}$. Assume that VD, (1.5), (1.6), $\mathrm{J}_{\phi, \leq}, \mathrm{FK}(\phi), \operatorname{PI}(\phi)$ and $\operatorname{CSJ}(\phi)$ hold. Suppose that there exist constants $\lambda>0$ and $\sigma \in(0,1]$ such that

$$
\mu\left(B_{r} \cap\{u \geq \lambda\}\right) \geq \sigma \mu\left(B_{r}\right)
$$

for some $r$ satisfying $0<r<R /(12 \kappa)$, where $\kappa \geq 1$ is the constant in $\operatorname{PI}(\phi)$. Then, there exists a constant $\delta \in(0,1 / 4)$ depending on $\sigma$ but independent of $\lambda, r, R, x_{0}$ and $u$, such that

$$
\operatorname{ess} \inf _{B_{4 r}} u \geq \delta \lambda-\phi\left(x_{0}, r\right) \operatorname{Tail}_{\phi}\left(u_{-} ; x_{0}, R\right) .
$$

Proof. Without loss of generality, we may and do assume that

$$
\phi\left(x_{0}, r\right) \operatorname{Tail}_{\phi}\left(u_{-} ; x_{0}, R\right) \leq \delta \lambda ;
$$

otherwise the conclusion is trivial due to the fact that $u \geq 0$ on $B_{R}$.

For any $j \geq 0$, define

$$
l_{j}=\delta \lambda+2^{-j-1} \delta \lambda, \quad r_{j}=4 r+2^{1-j} r .
$$

Then, by (3.5) we see that

$$
l_{0}=\frac{3}{2} \delta \lambda \leq 2 \delta \lambda-\frac{1}{2} \phi\left(x_{0}, r\right) \operatorname{Tail}_{\phi}\left(u_{-} ; x_{0}, R\right) .
$$

Lemma 3.3 implies that

$$
\frac{\mu\left(B_{6 r} \cap\left\{u \leq l_{0}\right\}\right)}{\mu\left(B_{6 r}\right)} \leq \frac{c_{1}}{\sigma \log \frac{1}{2 \delta}} .
$$

In the following, let us denote by

$$
B_{j}=B_{r_{j}}, \quad w_{j}=\left(l_{j}-u\right)_{+}, \quad A_{j}=\frac{\mu\left(B_{j} \cap\left\{u \leq l_{j}\right\}\right)}{\mu\left(B_{j}\right)} .
$$

Note that, $-u$ is an $\mathcal{E}$-subharmonic function on $B_{R}$. Then, we have by Lemma 3.4 that

$$
\begin{aligned}
& A_{j+2}\left(l_{j+1}-l_{j+2}\right)^{2} \\
&=\frac{1}{\mu\left(B_{j+2}\right)} \int_{B_{j+2} \cap\left\{u \leq l_{j+2}\right\}}\left(l_{j+1}-l_{j+2}\right)^{2} d \mu \\
& \leq \frac{1}{\mu\left(B_{j+2}\right)} \int_{B_{j+2}} w_{j+1}^{2} d \mu \\
& \leq \frac{c_{2}}{\left(l_{j}-l_{j+1}\right)^{2 \nu}}\left(\frac{1}{\mu\left(B_{j+1}\right)} \int_{B_{j+1}} w_{j}^{2} d \mu\right)^{1+\nu}\left(\frac{r_{j+2}}{r_{j+1}-r_{j+2}}\right)^{\beta_{2}} \\
& \quad \times\left[1+\frac{1}{l_{j}-l_{j+1}}\left(\frac{r_{j+2}}{r_{j+1}-r_{j+2}}\right)^{d_{2}+\beta_{2}-\beta_{1}} \phi\left(x_{0}, r_{j+1}\right) \operatorname{Tail}_{\phi}\left(w_{j} ; x_{0}, r_{j+1}\right)\right] \\
& \leq \frac{c_{3}}{\left[\left(2^{-j}-2^{-j-1}\right) \delta \lambda\right]^{2 \nu}}\left[(\delta \lambda)^{2} A_{j}\right]^{1+\nu}\left(\frac{1}{2^{-j}-2^{-j-1}}\right)^{\beta_{2}}
\end{aligned}
$$




$$
\begin{aligned}
& \times\left[1+\frac{1}{\left(2^{-j}-2^{-j-1}\right) \delta \lambda}\left(\frac{1}{2^{-j}-2^{-j-1}}\right)^{d_{2}+\beta_{2}-\beta_{1}} \phi\left(x_{0}, r_{j+1}\right) \operatorname{Tail}_{\phi}\left(w_{j} ; x_{0}, r_{j+1}\right)\right] \\
\leq & c_{4}(\delta \lambda)^{2} A_{j}^{1+\nu} 2^{\left(1+2 \nu+d_{2}+2 \beta_{2}-\beta_{1}\right) j}\left(1+\frac{1}{\delta \lambda} \phi\left(x_{0}, r_{j+1}\right) \operatorname{Tail}_{\phi}\left(w_{j} ; x_{0}, r_{j+1}\right)\right),
\end{aligned}
$$

where $\nu$ is the constant in $\mathrm{FK}(\phi)$, and in the third inequality we have used the facts that $w_{j} \leq l_{j} \leq 3 \delta \lambda / 2$ and

$$
\int_{B_{j+1}} w_{j}^{2} d \mu \leq c^{\prime}(\delta \lambda)^{2} \mu\left(B_{j+1} \cap\left\{w_{j} \geq 0\right\}\right) \leq c^{\prime \prime}(\delta \lambda)^{2} \mu\left(B_{j} \cap\left\{u \leq l_{j}\right\}\right) .
$$

Note that

$$
\begin{aligned}
& \phi\left(x_{0}, r_{j+1}\right) \operatorname{Tail}_{\phi}\left(w_{j} ; x_{0}, r_{j+1}\right) \\
& =\phi\left(x_{0}, r_{j+1}\right) \int_{B_{j+1}^{c}} \frac{\left|w_{j}\right|(z)}{V\left(x_{0}, d\left(x_{0}, z\right)\right) \phi\left(x_{0}, d\left(x_{0}, z\right)\right)} \mu(d z) \\
& \leq \phi\left(x_{0}, r_{j+1}\right) \int_{B_{j+1}^{c}} \frac{l_{j}+u_{-}(z)}{V\left(x_{0}, d\left(x_{0}, z\right)\right) \phi\left(x_{0}, d\left(x_{0}, z\right)\right)} \mu(d z) \\
& =\phi\left(x_{0}, r_{j+1}\right)\left[\int_{B_{R} \backslash B_{j+1}} \frac{l_{j}}{V\left(x_{0}, d\left(x_{0}, z\right)\right) \phi\left(x_{0}, d\left(x_{0}, z\right)\right)} \mu(d z)\right. \\
& \left.\quad+\int_{B_{R}^{c}} \frac{l_{j}+u_{-}(z)}{V\left(x_{0}, d\left(x_{0}, z\right)\right) \phi\left(x_{0}, d\left(x_{0}, z\right)\right)} \mu(d z)\right] \\
& \leq c_{5}\left(l_{j}+\frac{\phi\left(x_{0}, r_{j+1}\right)}{\phi\left(x_{0}, R\right)} l_{j}+\phi\left(x_{0}, r\right) \operatorname{Tail}_{\phi}\left(u_{-} ; x_{0}, R\right)\right) \\
& \leq c_{6} \delta \lambda,
\end{aligned}
$$

where in the second equality we have used the fact that $u \geq 0$ on $B_{R}$, in the second inequality we used Remark 1.5, and the last inequality follows from (3.5).

According to all the estimates above, we see that there is a constant $c_{7}>0$ such that for all $j \geq 0$,

$$
A_{j+2} \leq c_{7} A_{j}^{1+\nu} 2^{\left(3+2 \nu+d_{2}+2 \beta_{2}-\beta_{1}\right) j} .
$$

Let $c^{*}=c_{7}^{-1 / \nu} 2^{-\left(3+2 \nu+d_{2}+2 \beta_{2}-\beta_{1}\right) / \nu^{2}}$ and choose the constant $\delta \in(0,1 / 4)$ such that

$$
\frac{c_{1}}{\sigma \log \frac{1}{2 \delta}} \leq c^{*}
$$

then, by (3.6),

$$
A_{0} \leq c^{*}=c_{7}^{-1 / \nu} 2^{-\left(3+2 \nu+d_{2}+2 \beta_{2}-\beta_{1}\right) / \nu^{2}} .
$$

According to Lemma 3.5 , we can deduce that $\lim _{i \rightarrow \infty} A_{i}=0$. Therefore, $u \geq \delta \lambda$ on $B_{4 r}$, from which the desired assertion follows easily.

Remark 3.7. Proposition [3.6 contains GHH, Lemma 4.5] as a special case. Among other things, in [GHH, Lemma 4.5], it is assumed that $\phi(x, r)=r^{\alpha}$ for $\mathrm{J}_{\phi}$. Inequality (3.4) under condition (3.3) is called a weak Harnack inequality in [GHH]. 
Below is a Krylov-Safonov covering lemma on metric measure spaces, whose proof is essentially taken from $\left[\mathrm{KS}\right.$, Lemma 7.2]. Note that the difference of the following definition of $[E]_{\eta}$ from that in [KS, Lemma 7.2] is that here we impose the restriction of $0<\rho<r$ and change the constant 3 to 5 . For the sake of completeness, we present the proof here.

Lemma 3.8. Suppose that VD holds. Let $B_{r}\left(x_{0}\right)=B\left(x_{0}, r\right)$ for some $x_{0} \in M$ and any $r>0$. Let $E \subset B_{r}\left(x_{0}\right)$ be a measurable set. For any $\eta \in(0,1)$, define

$$
[E]_{\eta}=\bigcup_{0<\rho<r}\left\{B_{5 \rho}(x) \cap B_{r}\left(x_{0}\right): x \in B_{r}\left(x_{0}\right) \text { and } \frac{\mu\left(E \cap B_{5 \rho}(x)\right)}{\mu\left(B_{\rho}(x)\right)}>\eta\right\} .
$$

Then, either

$$
[E]_{\eta}=B_{r}\left(x_{0}\right)
$$

or

$$
\mu\left([E]_{\eta}\right) \geq \frac{1}{\eta} \mu(E)
$$

Proof. Define a maximal operator $A: B_{r}\left(x_{0}\right) \rightarrow[0, \infty)$ as follows

$$
A(x)=\sup _{y \in B_{r}\left(x_{0}\right), x \in B_{5 \rho}(y), 0<\rho<r} \frac{\mu\left(E \cap B_{5 \rho}(y)\right)}{\mu\left(B_{\rho}(y)\right)} .
$$

We claim that

$$
[E]_{\eta}=\left\{x \in B_{r}\left(x_{0}\right): A(x)>\eta\right\}
$$

for any $\eta \in(0,1)$. Indeed, let $x \in B_{r}\left(x_{0}\right)$ with $A(x)>\eta$. Then, there is a ball $B_{\rho}(y)$ with $0<\rho<r, y \in B_{r}\left(x_{0}\right)$ and $x \in B_{5 \rho}(y)$ such that $\frac{\mu\left(E \cap B_{5 \rho}(y)\right)}{\mu\left(B_{\rho}(y)\right)}>\eta$. This means that

$$
x \in\left\{B_{5 \rho}(y) \cap B_{r}\left(x_{0}\right): y \in B_{r}\left(x_{0}\right) \text { and } \frac{\mu\left(E \cap B_{5 \rho}(y)\right)}{\mu\left(B_{\rho}(y)\right)}>\eta\right\} \subset[E]_{\eta} .
$$

On the other hand, if $x \in[E]_{\eta}$, then there is a ball $B_{\rho}(y)$ with $0<\rho<r, y \in B_{r}\left(x_{0}\right)$ and $x \in B_{5 \rho}(y)$ such that $\frac{\mu\left(E \cap B_{5 \rho}(y)\right)}{\mu\left(B_{\rho}(y)\right)}>\eta$. This implies that $A(x)>\eta$.

Suppose that $B_{r}\left(x_{0}\right) \backslash[E]_{\eta} \neq \emptyset$. The set $[E]_{\eta}$ is open by definition. We cover $[E]_{\eta}$ by ball $B_{r_{x}}(x)$, where $x \in[E]_{\eta}$ and $r_{x}=d\left(x, B_{r}\left(x_{0}\right) \backslash[E]_{\eta}\right) / 2 \in(0, r)$. By the Vitali covering lemma 1, there are countably many pairwise disjoint balls $B_{r_{i}}\left(x_{i}\right)$, where $r_{i}=r_{x_{i}}$ for all $i \geq 1$, such that $[E]_{\eta} \subset \bigcup_{i=1}^{\infty} B_{5 r_{i}}\left(x_{i}\right)$. Note that, $B_{5 r_{i}}\left(x_{i}\right) \cap\left(B_{r}\left(x_{0}\right) \backslash[E]_{\eta}\right) \neq \emptyset$ for all $i \geq 1$, and so there is a point $y_{i} \in B_{5 r_{i}}\left(x_{i}\right) \cap\left(B_{r}\left(x_{0}\right) \backslash[E]_{\eta}\right)$. In particular, $A\left(y_{i}\right) \leq \eta$ for all $i \geq 1$. Since $y_{i} \in B_{r}\left(x_{0}\right)$, $x_{i} \in B_{5 r_{i}}\left(y_{i}\right)$ and $0<r_{i}<r$, we conclude that

$$
\mu\left(E \cap B_{5 r_{i}}\left(x_{i}\right)\right) \leq \eta \mu\left(B_{r_{i}}\left(x_{i}\right)\right) .
$$

If $y$ is the density point $E$ (i.e. $y \in E$ such that $\lim _{\rho \rightarrow 0} \frac{\mu\left(E \cap B_{\rho}(y)\right)}{\mu\left(B_{\rho}(y)\right)}=1$ ), then

$$
\liminf _{\rho \rightarrow 0} \frac{\mu\left(E \cap B_{5 \rho}(y)\right)}{\mu\left(B_{\rho}(y)\right)} \geq \lim _{\rho \rightarrow 0} \frac{\mu\left(E \cap B_{\rho}(y)\right)}{\mu\left(B_{\rho}(y)\right)}=1>\eta .
$$

\footnotetext{
${ }^{1}$ The VD condition implies a covering theorem, here referred to as the Vitali covering theorem. Indeed, given any collection of balls with uniformly bounded radius, there exists a pairwise disjoint, countable subcollection of balls, whose 5-dilates cover the union of the original collection. See [He, Chapter 1] for more details.
} 
Since $\mu$-almost every point $E$ is a density point; that is, for almost all $x \in E$, it holds that $\lim _{\rho \rightarrow 0} \frac{\mu\left(E \cap B_{\rho}(x)\right)}{\mu\left(B_{\rho}(x)\right)}=1$, which follows from VD and the Lebesgue differentiation theorem (see [He, Theorem 1.8]), we observe that $\mu$-almost every point of $E$ belongs to $[E]_{\eta}$ for every $\eta \in(0,1)$. From this it follows that

$$
\mu(E)=\mu\left(E \cap[E]_{\eta}\right) \leq \sum_{i=1}^{\infty} \mu\left(E \cap B_{5 r_{i}}\left(x_{i}\right)\right) \leq \eta \sum_{i=1}^{\infty} \mu\left(B_{r_{i}}\left(x_{i}\right)\right) \leq \eta \mu\left([E]_{\eta}\right) .
$$

The above inequality yields the desired result.

We now are in a position to present the

Proof of Theorem 3.1. Fix $\eta \in(0,1)$. Let us define for any $t>0$ and $i \geq 0$

$$
A_{t}^{i}=\left\{x \in B_{r}\left(x_{0}\right): u(x)>t \delta^{i}-\frac{T}{1-\delta}\right\},
$$

where $\delta \in(0,1)$ is determined later and

$$
T=\phi\left(x_{0}, 5 r\right) \operatorname{Tail}_{\phi}\left(u_{-} ; x_{0}, R\right) .
$$

Obviously we have $A_{t}^{i-1} \subset A_{t}^{i}$ for all $i \geq 1$. If there are a point $x \in B_{r}\left(x_{0}\right)$ and $0<\rho<r$ such that $B_{5 \rho}(x) \cap B_{r}\left(x_{0}\right) \subset\left[A_{t}^{i-1}\right]_{\eta}$, then, by the definition of $\left[A_{t}^{i-1}\right]_{\eta}$ and $\mathrm{VD}$,

$$
\mu\left(A_{t}^{i-1} \cap B_{5 \rho}(x)\right) \geq \eta \mu\left(B_{\rho}(x)\right) \geq c^{\prime} \eta \mu\left(B_{5 \rho}(x)\right),
$$

where $c^{\prime}$ is a positive constant independent of $\eta, x_{0}$ and $\rho$. Below, let $\delta$ be the constant given in Proposition 3.6 corresponding to the factor $c^{\prime} \eta$. Applying Proposition 3.6 with $\lambda=t \delta^{i-1}-\frac{T}{1-\delta}$ and $\sigma=c^{\prime} \eta$, we get that

$$
\begin{aligned}
\operatorname{ess}_{\inf }{ }_{B_{20 \rho}(x)} u & >\delta\left(t \delta^{i-1}-\frac{T}{1-\delta}\right)-\phi\left(x_{0}, 5 \rho\right) \operatorname{Tail}_{\phi}\left(u_{-} ; x_{0}, R\right) \\
& \geq \delta\left(t \delta^{i-1}-\frac{T}{1-\delta}\right)-T \\
& =t \delta^{i}-\frac{T}{1-\delta} .
\end{aligned}
$$

Hence, if $B_{5 \rho}(x)$ is one of the balls to make up to the set $\left[A_{t}^{i-1}\right]_{\eta}$ in Lemma 3.8, then $B_{5 \rho}(x) \cap$ $B_{r}\left(x_{0}\right) \subset A_{t}^{i}$, which implies that $\left[A_{t}^{i-1}\right]_{\eta} \subset A_{t}^{i}$. By Lemma 3.8, we must have either $A_{t}^{i}=B_{r}\left(x_{0}\right)$ (since $A_{t}^{i} \subset B_{r}\left(x_{0}\right)$ for all $i \geq 0$ ) or

$$
\mu\left(A_{t}^{i}\right) \geq \frac{1}{\eta} \mu\left(A_{t}^{i-1}\right)
$$

We choose an integer $j \geq 1$ so that

$$
\eta^{j}<\mu\left(A_{t}^{0}\right) / \mu\left(B_{r}\left(x_{0}\right)\right) \leq \eta^{j-1} .
$$

Suppose first that $A_{t}^{j-1} \neq B_{r}\left(x_{0}\right)$. Then, by the fact that $A_{t}^{i-1} \subset A_{t}^{i}$ for all $i \geq 1$, we have $A_{t}^{k} \neq B_{r}\left(x_{0}\right)$ for all $0 \leq k \leq j-1$. Hence, according to (3.7), we obtain that

$$
\mu\left(A_{t}^{j-1}\right) \geq \frac{1}{\eta} \mu\left(A_{t}^{j-2}\right) \geq \cdots \geq \frac{1}{\eta^{j-1}} \mu\left(A_{t}^{0}\right) \geq \eta \mu\left(B_{r}\left(x_{0}\right)\right) .
$$


Note that, the inequality holds trivially for the case that $A_{t}^{j-1}=B_{r}\left(x_{0}\right)$, thanks to the fact that $\eta \in(0,1)$. Therefore, according to Proposition 3.6 again, we have

$$
\begin{aligned}
\operatorname{ess~inf~}_{B_{4 r}\left(x_{0}\right)} u & \geq c_{1}\left(t \delta^{j-1}-\frac{T}{1-\delta}\right)-T \\
& \geq c_{1} t \delta^{j-1}-\frac{c_{2} T}{1-\delta} \\
& \geq c_{1} t\left(\frac{\mu\left(A_{t}^{0}\right)}{\mu\left(B_{r}\left(x_{0}\right)\right)}\right)^{1 / \gamma}-\frac{c_{2} T}{1-\delta},
\end{aligned}
$$

where $c_{1}$ is the constant given in Proposition 3.6 corresponding to the factor $\delta$, and $\gamma=\log _{\delta} \eta$. This is,

$$
\frac{\mu\left(A_{t}^{0}\right)}{\mu\left(B_{r}\left(x_{0}\right)\right)} \leq \frac{c_{3}}{t^{\gamma}}\left(\operatorname{ess~inf}_{B_{4 r}\left(x_{0}\right)} u+\frac{T}{1-\delta}\right)^{\gamma} .
$$

By Cavalieri's principle, we have for any $0<\varepsilon<\gamma$ and $a>0$,

$$
\begin{aligned}
\frac{1}{\mu\left(B_{r}\left(x_{0}\right)\right)} \int_{B_{r}\left(x_{0}\right)} u^{\epsilon} d \mu & =\varepsilon \int_{0}^{\infty} t^{\varepsilon-1} \frac{\mu\left(B_{r}\left(x_{0}\right) \cap\{u>t\}\right)}{\mu\left(B_{r}\left(x_{0}\right)\right)} d t \\
& \leq \varepsilon \int_{0}^{\infty} t^{\varepsilon-1} \frac{\mu\left(A_{t}^{0}\right)}{\mu\left(B_{r}\left(x_{0}\right)\right)} d t \\
& \leq \varepsilon\left[\int_{0}^{a} t^{\varepsilon-1} d t+c_{3}\left(\operatorname{ess~inf}_{B_{4 r}\left(x_{0}\right)} u+\frac{T}{1-\delta}\right)^{\gamma} \int_{a}^{\infty} t^{\varepsilon-1-\gamma} d t\right] \\
& \leq c_{4}\left[a^{\varepsilon}+\left(\operatorname{essinf}_{B_{4 r}\left(x_{0}\right)} u+\frac{T}{1-\delta}\right)^{\gamma} a^{\varepsilon-\gamma}\right] .
\end{aligned}
$$

In particular, taking

$$
a=\operatorname{ess~inf}_{B_{4 r}\left(x_{0}\right)} u+\frac{T}{1-\delta},
$$

we finally get that

$$
\frac{1}{\mu\left(B_{r}\left(x_{0}\right)\right)} \int_{B_{r}\left(x_{0}\right)} u^{\epsilon} d \mu \leq c_{4}\left(\operatorname{ess~inf}_{B_{4 r}\left(x_{0}\right)} u+\frac{T}{1-\delta}\right)^{\varepsilon} .
$$

This along with (1.5) concludes the proof.

Remark 3.9. $\mathrm{WEHI}^{+}(\phi)$ is equivalent to the inequality (3.4) under VD and condition (3.3). Indeed, the proof above shows that (3.4) under $\mathrm{VD}$ and condition (3.3) implies $\mathrm{WEHI}^{+}(\phi)$. Conversely, assume $\mathrm{WEHI}^{+}(\phi)$ holds. Under condition (3.3) we have

$$
\left(\frac{1}{\mu\left(B_{r}\right)} \int_{B_{r}} u^{\varepsilon} d \mu\right)^{1 / \varepsilon} \geq\left(\frac{1}{\mu\left(B_{r}\right)} \int_{B_{r} \cap\{u \geq \lambda\}} u^{\varepsilon} d \mu\right)^{1 / \varepsilon} \geq \lambda \sigma^{1 / \varepsilon} .
$$

Plugging this into $\mathrm{WEHI}^{+}(\phi)$ yields (3.4).

\section{Implications of EHI}

In this section, we first study the relation between $\mathrm{EHI}$ and $\operatorname{EHI}(\phi)$, and then show that under some conditions EHI implies $\mathrm{PI}(\phi)$. 


\section{$4.1 \quad \mathrm{EHI}+\mathrm{E}_{\phi, \leq}+\mathrm{J}_{\phi, \leq} \Longrightarrow \mathrm{EHI}(\phi)+\mathrm{FK}(\phi)$}

We first recall the following Lévy system formula. See, for example [CK2, Appendix A] for a proof.

Lemma 4.1. Let $f$ be a non-negative measurable function on $\mathbb{R}_{+} \times M \times M$ that vanishes along the diagonal. Then for every $t \geq 0, x \in M_{0}$ and stopping time $T$ (with respect to the filtration of $\left\{X_{t}\right\}$ ),

$$
\mathbb{E}^{x}\left[\sum_{s \leq T} f\left(s, X_{s-}, X_{s}\right)\right]=\mathbb{E}^{x}\left[\int_{0}^{T} \int_{M} f\left(s, X_{s}, y\right) J\left(X_{s}, d y\right) d s\right]
$$

For any open subset $D \subset M$, denote the transition semigroup of the part process $X^{D}=\left\{X_{t}^{D}\right\}$ of $X$ killed upon leaving $D$ by $\left\{P_{t}^{D}\right\}$; that is, for any $f \in \mathcal{B}_{+}(D)$,

$$
P_{t}^{D} f(x)=\mathbb{E}^{x}\left[f\left(X_{t}^{D}\right)\right]=\mathbb{E}^{x}\left[f\left(X_{t}\right) ; t<\tau_{D}\right], \quad x \in D \cap M_{0} .
$$

The Green operator $G_{D}$ is defined by

$$
G_{D} f(x)=\int_{0}^{\infty} P_{t}^{D} f(x) d t=\mathbb{E}^{x}\left[\int_{0}^{\tau_{D}} f\left(X_{t}\right) d t\right], \quad x \in D \cap M_{0} .
$$

For $f \in \mathcal{B}(D)$, if $P_{t}^{D}|f|(x)<\infty$, we define

$$
P_{t}^{D} f(x)=P_{t}^{D} f^{+}(x)-P_{t}^{D} f^{-}(x)=\mathbb{E}^{x}\left[f\left(X_{t}^{D}\right)\right] ;
$$

if $G_{D}|f|(x)<\infty$, we define

$$
G_{D} f(x)=G_{D} f^{+}(x)-G_{D} f^{-}(x)=\mathbb{E}^{x}\left[\int_{0}^{\tau_{D}} f\left(X_{t}\right) d t\right] .
$$

It is known that $\left\{P_{t}^{D}\right\}$ is the semigroup associated with the part Dirichlet form $\left(\mathcal{E}, \mathcal{F}_{D}\right)$ of $(\mathcal{E}, \mathcal{F})$ on $D$, where $\mathcal{F}_{D}$ is the $\sqrt{\mathcal{E}_{1}}$-completion of $\mathcal{F} \cap C_{c}(D)$. Let $\mathcal{L}_{D}$ be the $L^{2}$-generator of $\left(\mathcal{E}, \mathcal{F}_{D}\right)$ on $L^{2}(D ; \mu)$. The principle eigenvalue $\lambda_{1}(D)$ of $-\mathcal{L}_{D}$ is defined to be

$$
\lambda_{1}(D):=\inf \left\{\mathcal{E}(f, f): f \in \mathcal{F}_{D} \text { with }\|f\|_{L^{2}(D ; \mu)}=1\right\} .
$$

The following two lemmas are known, see [GT, Lemma 3.2] and [GH2, Lemma 5.1] respectively.

Lemma 4.2. If $\sup _{y \in D \backslash \mathcal{N}} \mathbb{E}^{y} \tau_{D}<\infty$, then $G_{D}$ is a bounded operator on $\mathcal{B}_{b}(D)$, and it uniquely extends to the space $L^{p}(D ; \mu)$ with $p=1,2, \infty$ and enjoys the following norm estimate

$$
\left\|G_{D}\right\|_{L^{p}(D ; \mu) \rightarrow L^{p}(D ; \mu)} \leq \sup _{y \in D \backslash \mathcal{N}} \mathbb{E}^{y} \tau_{D}
$$

Moreover, $G_{D}$ is the reverse of the operator $-\mathcal{L}_{D}$ in $L^{2}(D ; \mu)$, and

$$
\lambda_{1}(D)^{-1} \leq \sup _{y \in D \backslash \mathcal{N}} \mathbb{E}^{y} \tau_{D}
$$

Lemma 4.3. Let $(\mathcal{E}, \mathcal{F})$ be a regular Dirichlet form in $L^{2}(M ; \mu)$, and $D \subset M$ an open set such that $\lambda_{1}(D)>0$. Then the following hold: 
(i) For any $f \in L^{2}(D ; \mu)$,

$$
\left\|G_{D} f\right\|_{2} \leq \lambda_{1}(D)^{-1}\|f\|_{2} .
$$

(ii) For any $f \in L^{2}(D ; \mu), P_{t}^{D} f \in \mathcal{F}_{D}$ for every $t>0$ and $G_{D} f \in \mathcal{F}_{D}$. Moreover,

$$
\mathcal{E}\left(G_{D} f, g\right)=\langle f, g\rangle \quad \text { for every } g \in \mathcal{F}_{D} .
$$

Theorem 4.4. Assume that VD, (1.5), (1.6), $\mathrm{E}_{\phi, \leq}$ and $\mathrm{J}_{\phi, \leq}$ hold. Then $\mathrm{EHI}$ implies $\mathrm{EHI}(\phi)$.

Proof. Fix $x_{0} \in M$, and assume that $u$ is harmonic on $B_{R}:=B\left(x_{0}, R\right)$ such that $u \geq 0$ on $B_{R}$. Let $\delta \in(0,1)$ be the constant in EHI. Then, for almost all $x, y \in B_{\delta r}$ with $0<r \leq \delta R$,

$$
\begin{aligned}
u(x) & =\mathbb{E}^{x} u\left(X_{\tau_{B_{r}}}\right) \leq \mathbb{E}^{x} u_{+}\left(X_{\tau_{B_{r}}}\right) \leq c \mathbb{E}^{y} u_{+}\left(X_{\tau_{B_{r}}}\right) \\
& =c\left(\mathbb{E}^{y} u\left(X_{\tau_{B_{r}}}\right)+\mathbb{E}^{y} u_{-}\left(X_{\tau_{B_{r}}}\right)\right) \\
& =c u(y)+c \mathbb{E}^{y} u_{-}\left(X_{\tau_{B_{r}}}\right),
\end{aligned}
$$

where in the second inequality we used EHI. Since $u_{-}=0$ on $B_{R}$, by the Lévy system of $X$ and condition $\mathrm{J}_{\phi, \leq}$, we have for $y \in B_{\delta r}$

$$
\begin{aligned}
\mathbb{E}^{y} u_{-}\left(X_{\tau_{B_{r}}}\right) & =\mathbb{E}^{y}\left[\int_{0}^{\tau_{B_{r}}} \int_{B_{R}^{c}} u_{-}(z) J\left(X_{s}, z\right) \mu(d z) d s\right] \\
& =\int_{B_{R}^{c}} u_{-}(z)\left(G_{B_{r}} J(\cdot, z)\right)(y) \mu(d z) \\
& \leq c_{1} \int_{B_{R}^{c}} u_{-}(z)\left(G_{B_{r}} \frac{1}{V(\cdot, d(\cdot, z)) \phi(\cdot, d(\cdot, z))}\right)(y) \mu(d z) \\
& \leq c_{2} \int_{B_{R}^{c}} \frac{u_{-}(z)}{V\left(x_{0}, d\left(x_{0}, z\right)\right) \phi\left(x_{0}, d\left(x_{0}, z\right)\right)} \mu(d z) G_{B_{r}} \mathbf{1}(y) \\
& =c_{2} \int_{B_{R}^{c}} \frac{u_{-}(z)}{V\left(x_{0}, d\left(x_{0}, z\right)\right) \phi\left(x_{0}, d\left(x_{0}, z\right)\right)} \mu(d z) \mathbb{E}^{y} \tau_{B_{r}} \\
& \leq c_{3} \phi\left(x_{0}, r\right) \int_{B_{R}^{c}} \frac{u_{-}(z)}{V\left(x_{0}, d\left(x_{0}, z\right)\right) \phi\left(x_{0}, d\left(x_{0}, z\right)\right)} \mu(d z) \\
& =c_{3} \phi\left(x_{0}, r\right) \operatorname{Tail}_{\phi}\left(u_{-} ; x_{0}, R\right),
\end{aligned}
$$

where the second inequality follows from VD, (1.5) and (1.6), and in the last inequality we have used $\mathrm{E}_{\phi, \leq}$ and (1.6).

From Theorem 4.4, we can deduce the following.

Proposition 4.5. (i) Assume that VD, (1.5) and (1.6) hold. Then,

$$
\mathrm{EHR}+\mathrm{E}_{\phi, \leq} \Longrightarrow \mathrm{FK}(\phi) .
$$

(ii) Assume that $\mathrm{VD}$, (1.5), (1.6), $\mathrm{E}_{\phi, \leq}$ and $\mathrm{J}_{\phi, \leq}$ hold. Then $\mathrm{EHI}$ implies $\mathrm{FK}(\phi)$.

Proof. The first required assertion follows from the argument of [CKW2, Lemma 4.6]. By Theorems 4.4 and 2.1, we have EHI $+\mathrm{E}_{\phi, \leq}+\mathrm{J}_{\phi, \leq} \Longrightarrow$ EHR, which along with the first assertion immediately yields the second one. 


\section{$4.2 \mathrm{EHI}+\mathrm{E}_{\phi}+\mathrm{J}_{\phi, \leq} \Longrightarrow \mathrm{PI}(\phi)$}

Proposition 4.6. (i) Suppose that VD, (1.5), (1.6), EHR, $\mathrm{E}_{\phi}$ and $\mathrm{FK}(\phi)$ hold. Then $\mathrm{PI}(\phi)$ holds.

(ii) Suppose that VD, (1.5), (1.6), EHI, $\mathrm{E}_{\phi}$ and $\mathrm{J}_{\phi, \leq}$ hold. Then $\mathrm{PI}(\phi)$ holds.

Proof. By Theorems 4.4, 2.1 and Proposition 4.5, we have EHI $+\mathrm{E}_{\phi, \leq}+\mathrm{J}_{\phi, \leq} \Longrightarrow \mathrm{EHR}+\mathrm{FK}(\phi)$, so it is enough to prove (i). According to the proofs of [GH1, Lemmas 5.4 and 5.5] and choosing $a=C V(x, r)^{\nu} / \phi(x, r)$ in that paper, we can get from $\operatorname{FK}(\phi)$ that for any ball $B=B(x, r)$ with $x \in M$ and $r>0$, the Dirichlet heat kernel $p^{B}(t, x, y)$ exists, and there exists a constant $C_{1}>0$ such that

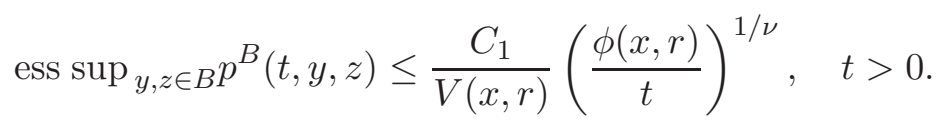

Using this estimate, $\mathrm{J}_{\phi,} \leq$ and EHR, and following the argument of [CKW2, Lemma 4.8], there are constants $\kappa, C_{2}>0$ such that for any $x \in M_{0}, t>0,0<r \leq 2^{-\left(\beta_{1}+\theta\right) / \beta_{1}} \phi^{-1}(x, t)$ and $y \in B(x, r) \backslash \mathcal{N}$,

$$
\left|p^{B\left(x, \phi^{-1}(x, t)\right)}(t, x, x)-p^{B\left(x, \phi^{-1}(x, t)\right)}(t, x, y)\right| \leq\left(\frac{r}{\phi^{-1}(x, t)}\right)^{\kappa} \frac{C_{2}}{V\left(x, \phi^{-1}(x, t)\right)},
$$

where $\beta_{1}$ is the constant in (1.5) and $\theta$ is the Hölder exponent in EHR.

Since for any $t>0, \tau_{B(x, r)} \leq t+\left(\tau_{B(x, r)}-t\right) \mathbf{1}_{\left\{\tau_{B(x, r)} \geq t\right\}}$, we have, by the Markov property, $\mathrm{E}_{\phi, \leq}$ and (1.6),

$$
\begin{aligned}
\mathbb{E}^{x} \tau_{B(x, r)} & \leq t+\mathbb{E}^{x}\left[\mathbf{1}_{\left\{\tau_{B(x, r)}>t\right\}} \mathbb{E}^{X{ }_{t}}\left[\tau_{B(x, r)}-t\right]\right] \leq t+\mathbb{P}^{x}\left(\tau_{B(x, r)}>t\right) \sup _{z \in B(x, r)} \mathbb{E}^{z} \tau_{B(x, r)} \\
& \leq t+\mathbb{P}^{x}\left(\tau_{B(x, r)}>t\right) \sup _{z \in B(x, r)} \mathbb{E}^{z} \tau_{B(z, 2 r)} \leq t+c_{2} \mathbb{P}^{x}\left(\tau_{B(x, r)}>t\right) \phi(x, r) .
\end{aligned}
$$

Then, by $\mathrm{E}_{\phi}$, for all $x \in M_{0}$,

$$
c_{1} \phi(x, r) \leq \mathbb{E}^{x} \tau_{B(x, r)} \leq t+c_{2} \mathbb{P}^{x}\left(\tau_{B(x, r)}>t\right) \phi(x, r)
$$

and so

$$
\mathbb{P}^{x}\left(\tau_{B(x, r)} \leq t\right) \leq 1-\frac{c_{1} \phi(x, r)-t}{c_{2} \phi(x, r)} .
$$

In particular, we can choose a constant $\delta>0$ such that for all $r>0$ and all $x \in M_{0}$,

$$
\mathbb{P}^{x}\left(\tau_{B(x, r)} \leq \delta \phi(x, r)\right) \leq 1-\frac{c_{1}}{2 c_{2}} .
$$

Combining with all the conclusions above, we can see from the argument of CKW2, Proposition 4.9] that there exist $\varepsilon \in(0,1)$ and $c_{3}>0$ such that for any $x_{0} \in M, r>0,0<t \leq \phi\left(x_{0}, \varepsilon r\right)$ and $B=B\left(x_{0}, r\right)$,

$$
p^{B}(t, x, y) \geq \frac{c_{3}}{V\left(x_{0}, \phi^{-1}\left(x_{0}, t\right)\right)}, \quad x, y \in B\left(x_{0}, \varepsilon \phi^{-1}\left(x_{0}, t\right)\right) \cap M_{0},
$$

which yields $\mathrm{PI}(\phi)$ by some standard arguments, see [CKW2, Proposition 3.5(i)].

At the end of this section, we present a consequence of $\mathrm{E}_{\phi}$ and $\mathrm{J}_{\phi, \leq}$ (without EHI). 
Proposition 4.7. Under VD, (1.5) and (1.6), $\mathrm{E}_{\phi}$ and $\mathrm{J}_{\phi, \leq}$ imply $\operatorname{CSJ}(\phi)$.

Proof. As shown in (4.1), under VD, (1.5) and (1.6), $\mathrm{E}_{\phi}$ implies that there are constants $\delta_{0}>0$ and $0<\varepsilon_{0}<1$ such that for all $r>0$ and all $x \in M_{0}$,

$$
\mathbb{P}^{x}\left(\tau_{B(x, r)} \leq \delta_{0} \phi(x, r)\right) \leq \varepsilon_{0} .
$$

Having this estimate at hand with $\mathrm{J}_{\phi, \leq}$, and following the arguments in [CKW1, Subsection 3.2] by replacing $\phi(r)$ with $\phi\left(x_{0}, r\right)$, we can prove the desired assertion.

\section{$5 \quad$ Exit time and relative capacity}

In this section, we study the relation between two-sided mean exit time estimates and two-sided relative capacitary estimates.

\subsection{From mean exit time estimates $\mathrm{E}_{\phi}$ to relative capacitary estimates}

Recall that for open subsets $A$ and $B$ of $M$ with $A \Subset B$, we define

$$
\operatorname{Cap}(A, B)=\inf \left\{\mathcal{E}(u, u): u \in \mathcal{F}, u=1 \mathcal{E} \text {-q.e. on } A \text { and } u=0 \mathcal{E} \text {-q.e. on } B^{c}\right\} \text {. }
$$

Note that $\operatorname{Cap}(A, B)$ is increasing in $A$ but decreasing in $B$.

Proposition 5.1. Under VD, (1.5) and (1.6), if $\mathrm{E}_{\phi}$ holds, then for any $B_{r}=B\left(x_{0}, r\right)$ with some $x_{0} \in M$ and $r>0$,

$$
\operatorname{Cap}\left(B_{r / 2}, B_{r}\right) \asymp \frac{V\left(x_{0}, r\right)}{\phi\left(x_{0}, r\right)} .
$$

Proof. Throughout the proof, define $g(x)=\mathbb{E}^{x} \tau_{B_{r}}=G_{B_{r}}$ 1. Set

$$
u=\frac{g}{\inf _{B_{r / 2}} g} \wedge 1 \text {. }
$$

Then, $\left.u\right|_{B_{r / 2}}=1,\left.u\right|_{B_{r}^{c}}=0$ and

$$
\mathcal{E}(u, u) \leq \frac{1}{\left(\inf _{B_{r / 2}} g\right)^{2}} \mathcal{E}(g, g)=\frac{\int_{B_{r}} g d \mu}{\left(\inf _{B_{r / 2}} g\right)^{2}} \leq \frac{c_{1} V\left(x_{0}, r\right)}{\phi\left(x_{0}, r\right)},
$$

where the equality follows from the fact that $\mathcal{L}_{B_{r}} g=-1$, and in the second inequality we have used $\mathrm{E}_{\phi}$, (1.5) and (1.6). Hence,

$$
\operatorname{Cap}\left(B_{r / 2}, B_{r}\right) \leq \mathcal{E}(u, u) \leq \frac{c_{1} V\left(x_{0}, r\right)}{\phi\left(x_{0}, r\right)} .
$$

On the other hand, since

$$
\frac{\left(\int_{B_{r}} g d \mu\right)^{2}}{\mathcal{E}(g, g)}=\frac{\left(\int_{B_{r}} g d \mu\right)^{2}}{\int_{B_{r}} g d \mu}=\int_{B_{r}} g d \mu
$$


and for any $u \in \mathcal{F}_{B_{r}}$

$$
\int_{B_{r}} u d \mu=\mathcal{E}\left(u, G_{B_{r}} \mathbf{1}\right) \leq \sqrt{\mathcal{E}(u, u)} \sqrt{\mathcal{E}\left(G_{B_{r}} \mathbf{1}, G_{B_{r}} \mathbf{1}\right)}=\sqrt{\mathcal{E}(u, u)} \sqrt{\int_{B_{r}} g d \mu,}
$$

we have

$$
\int_{B_{r}} g d \mu=\sup \left\{\frac{\left(\int_{B_{r}} u d \mu\right)^{2}}{\mathcal{E}(u, u)}: u \in \mathcal{F}_{B_{r}}\right\} .
$$

Applying the inequality above with $\mathrm{E}_{\phi}, \mathrm{VD}$, (1.5) and (1.6), we find that

$$
\begin{aligned}
c_{2} V\left(x_{0}, r\right) \phi\left(x_{0}, r\right) & \geq \int_{B_{r}} g d \mu=\sup \left\{\frac{\left(\int_{B_{r}} u d \mu\right)^{2}}{\mathcal{E}(u, u)}: u \in \mathcal{F}_{B_{r}}\right\} \\
& \geq V\left(x_{0}, r / 2\right)^{2} \sup \left\{\frac{1}{\mathcal{E}(u, u)}: u \in \mathcal{F}_{B_{r}},\left.u\right|_{B_{r / 2}}=1\right\} \\
& =\frac{c_{3} V\left(x_{0}, r\right)^{2}}{\operatorname{Cap}\left(B_{r / 2}, B_{r}\right)} .
\end{aligned}
$$

Hence,

$$
\operatorname{Cap}\left(B_{r / 2}, B_{r}\right) \geq \frac{c_{4} V\left(x_{0}, r\right)}{\phi\left(x_{0}, r\right)}
$$

The proof is complete.

\subsection{From capacitary estimates to mean exit time estimates $\mathrm{E}_{\phi}$}

In this subsection, we assume that Assumptions 1.13 and 1.14 hold, and will prove that

$$
\mathrm{EHI}+\mathrm{J}_{\mathrm{Ext}, \leq} \Longrightarrow \mathrm{E}_{\mathrm{Ext}},
$$

which will yield Theorem 1.16. Recall that the RVD condition (1.3) is equivalent to the existence of $l_{\mu}>1$ and $\widetilde{c}_{\mu}>1$ so that

$$
V\left(x, l_{\mu} r\right) \geq \widetilde{c}_{\mu} V(x, r) \quad \text { for all } x \in M \text { and } r>0,
$$

which implies that

$$
\mu\left(B\left(x, l_{\mu} r\right) \backslash B(x, r)\right)>0 \quad \text { for each } x \in M \text { and } r>0 .
$$

For any set $A \subset M$, define its first hitting time $\sigma_{A}:=\inf \left\{t>0: X_{t} \in A\right\}$. Recall that for two relatively compact open sets $A$ and $B$ of $M$ with $A \Subset B, \operatorname{Cap}(A, B)$ is the 0-order capacity in the transient Dirichlet form $\left(\mathcal{E}, \mathcal{F}_{B}\right)$, which is the Dirichlet form in $L^{2}(B ; \mu)$ of the subprocess $X^{B}$ of the symmetric Hunt process $X$ killed upon leaving $B$. Thus there exists a unique smooth measure $\nu:=\nu_{A, B}$ with $\operatorname{supp}[\nu] \subset \bar{A}$ such that $G_{B} \nu$ is the 0-order equilibrium potential of $A$ in $\left(\mathcal{E}, \mathcal{F}_{B}\right)$ and $\operatorname{Cap}(A, B)=\nu(\bar{A})$, see e.g. [CF, Page 87-88 and (3.4.3)] or the 0 -order version of [FOT, (2.2.13)]. Here $G_{B} \nu(x):=\int_{B} G_{B}(x, y) \nu(d y)$. This measure $\nu$ is called the relative capacitary measure of $A$ in $B$. In particular, according to [CF, Corollary 3.4.3] or [FOT, Theorem 4.3.3],

$$
G_{B} \nu(x)=\mathbb{P}^{x}\left(\sigma_{A}<\tau_{B}\right) \text { for } \mathcal{E} \text {-q.e. } x \in M \text {. }
$$


Lemma 5.2. Assume that Assumptions 1.13, 1.14 and $\mathrm{EHI}$ hold. Then $\mathrm{E}_{\mathrm{Ext}, \leq}$ holds; that is, there is a constant $c_{1}>0$ such that for almost all $x \in M$ and any $r>0$,

$$
\mathbb{E}^{x} \tau_{B(x, r)} \leq c_{1} \operatorname{Ext}(x, r)
$$

Proof. For $x_{0} \in M$ and $r>0$, let $D=B\left(x_{0}, 2 r\right)$. Let $\delta \in(0,1)$ be the constant in EHI. For any $y \in B\left(x_{0}, r\right) \backslash B\left(x_{0}, r / 2\right)$, let $\nu$ be the relative capacitary measure for $B(y, \delta r / 3)$ with respect to $G_{D}(x, y)$. Then $\operatorname{supp}(\nu) \subset \overline{B(y, \delta r / 3)}$, where $\bar{A}$ denotes the closure of the set $A$ in $M$. Applying EHI for $G_{D}\left(x_{0}, \cdot\right)$ on $B(y, r / 2)$, we have

$$
\begin{aligned}
1 & \geq \mathbb{P}^{x_{0}}\left(\sigma_{B(y, \delta r / 3)}<\tau_{D}\right)=\int \frac{}{B(y, \delta r / 3)} G_{D}\left(x_{0}, z\right) \nu(d z) \asymp G_{D}\left(x_{0}, y\right) \nu(\overline{B(y, \delta r / 3)}) \\
& =G_{D}\left(x_{0}, y\right) \operatorname{Cap}\left(B(y, \delta r / 3), B\left(x_{0}, 2 r\right)\right) \geq G_{D}\left(x_{0}, y\right) \operatorname{Cap}(B(y, \delta r / 3), B(y, 4 r)) \\
& \asymp G_{D}\left(x_{0}, y\right) \operatorname{Cap}\left(B\left(x_{0}, r\right), B\left(x_{0}, 2 r\right)\right),
\end{aligned}
$$

where the second inequality follows from the facts that $B\left(x_{0}, 2 r\right) \subset B(y, 4 r)$ and $\operatorname{Cap}(A, B)$ is decreasing in $B$, and in the last step we used (1.10). Thus,

$$
G_{B\left(x_{0}, 2 r\right)}\left(x_{0}, y\right) \leq \frac{c_{1}}{\operatorname{Cap}\left(B\left(x_{0}, r\right), B\left(x_{0}, 2 r\right)\right)} \quad \text { for all } y \in B\left(x_{0}, r\right) \backslash B\left(x_{0}, r / 2\right) .
$$

In particular,

$$
\begin{aligned}
\int_{B\left(x_{0}, r\right)} G_{B\left(x_{0}, 2 r\right)}\left(x_{0}, y\right) \mu(d y) \leq & \int_{B\left(x_{0}, r / 2\right)} G_{B\left(x_{0}, 2 r\right)}(x, y) \mu(d y) \\
& +\frac{c_{2} V\left(x_{0}, r\right)}{\operatorname{Cap}\left(B\left(x_{0}, r\right), B\left(x_{0}, 2 r\right)\right)} .
\end{aligned}
$$

On the other hand, by the strong Markov property of $X^{B\left(x_{0}, 2 r\right)}$, for every $y \in B\left(x_{0}, r\right)$,

$$
\begin{aligned}
G_{B\left(x_{0}, 2 r\right)}\left(x_{0}, y\right) & =G_{B\left(x_{0}, r\right)}\left(x_{0}, y\right)+\mathbb{E}^{y}\left[G_{B\left(x_{0}, 2 r\right)}\left(x_{0}, X_{\left.\tau_{B\left(x_{0}, r\right)}\right)}\right) ; \tau_{B\left(x_{0}, r\right)}<\tau_{B\left(x_{0}, 2 r\right)}\right] \\
& \leq G_{B\left(x_{0}, r\right)}\left(x_{0}, y\right)+\mathbb{E}^{y}\left[G_{B\left(x_{0}, 4 r\right)}\left(x_{0}, X_{\left.\tau_{B\left(x_{0}, r\right)}\right)}\right) ; \tau_{B\left(x_{0}, r\right)}<\tau_{B\left(x_{0}, 2 r\right)}\right] \\
& \leq G_{B\left(x_{0}, r\right)}\left(x_{0}, y\right)+\frac{c_{1}}{\operatorname{Cap}\left(B\left(x_{0}, 2 r\right), B\left(x_{0}, 4 r\right)\right)} \\
& \leq G_{B\left(x_{0}, r\right)}\left(x_{0}, y\right)+\frac{c_{3}}{\operatorname{Cap}\left(B\left(x_{0}, r\right), B\left(x_{0}, 2 r\right)\right)}
\end{aligned}
$$

where in the second and the third inequalities, we used (5.3) and (1.10), respectively. Hence,

$$
\int_{B\left(x_{0}, r / 2\right)} G_{B\left(x_{0}, 2 r\right)}\left(x_{0}, y\right) \mu(d y) \leq \int_{B\left(x_{0}, r / 2\right)} G_{B\left(x_{0}, r\right)}\left(x_{0}, y\right) \mu(d y)+\frac{c_{4} V\left(x_{0}, r\right)}{\operatorname{Cap}\left(B\left(x_{0}, r\right), B\left(x_{0}, 2 r\right)\right)} .
$$

This together with (5.4) yields that

$$
\begin{aligned}
\int_{B\left(x_{0}, r\right)} G_{B\left(x_{0}, 2 r\right)}\left(x_{0}, y\right) \mu(d y) & \leq \int_{B\left(x_{0}, r / 2\right)} G_{B\left(x_{0}, r\right)}\left(x_{0}, y\right) \mu(d y)+\frac{c_{5} V\left(x_{0}, r\right)}{\operatorname{Cap}\left(B\left(x_{0}, r\right), B\left(x_{0}, 2 r\right)\right)} \\
& =\int_{B\left(x_{0}, r / 2\right)} G_{B\left(x_{0}, r\right)}\left(x_{0}, y\right) \mu(d y)+c_{5} \operatorname{Ext}\left(x_{0}, r\right) .
\end{aligned}
$$


By iterating the above estimate and using the reverse doubling property of $\operatorname{Ext}\left(x_{0}, r\right)$ in Assumption 1.13(iv), we obtain that

$$
\begin{aligned}
\mathbb{E}^{x_{0}} \tau_{B\left(x_{0}, r\right)} & \leq \int_{B\left(x_{0}, r\right)} G_{B\left(x_{0}, 2 r\right)}\left(x_{0}, y\right) \mu(d y) \leq c_{5} \sum_{k=0}^{\infty} \operatorname{Ext}\left(x_{0}, 2^{-k} r\right) \\
& \leq c_{5} \operatorname{Ext}\left(x_{0}, r\right) \sum_{k=0}^{\infty} 2^{-k \beta_{1}}=c_{6} \operatorname{Ext}\left(x_{0}, r\right),
\end{aligned}
$$

where the second inequality is due to $\mathbb{E}^{x_{0}}\left[\int_{0}^{\tau_{D}} \mathbf{1}_{\left\{x_{0}\right\}}\left(X_{t}\right) d t\right]=0$, which is a consequence of Assumption 1.14. The proof is complete.

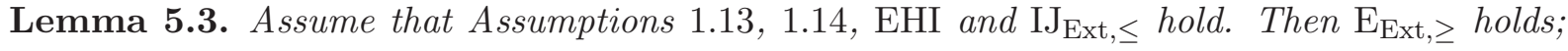
that is, there exists a constant $c_{1}>0$ such that almost all $x \in M$ and any $r>0$,

$$
\mathbb{E}^{x} \tau_{B(x, r)} \geq c_{1} \operatorname{Ext}(x, r)
$$

Proof. First note that by (5.2), $\tau_{B(x, r)}<\infty$ a.s. $\mathbb{P}^{x}$. Next by (1.9), we can choose $l \geq 3 \vee l_{\mu}$ large enough, where $l_{\mu}$ is in (5.1), such that there is a constant $\rho \in(0,1)$ so that

$$
\operatorname{Ext}(x, r) \leq \rho \operatorname{Ext}(x, l r) \quad \text { for every } x \in M \text { and } r>0
$$

Let $D=B\left(x, l^{k+1} r\right)$ where $k \geq 1$ will be determined later. Note that the set $B\left(x, l^{k} r\right) \backslash B(x, r)$ is non-empty due to (5.1). According to the Lévy system, for fixed $x \in M_{0}$,

$$
\begin{aligned}
\mathbb{P}^{x}\left(\sigma_{B\left(x, l^{k} r\right) \backslash B(x, r)}<\tau_{D}\right) & \geq \mathbb{P}^{x}\left(X_{\tau_{B(x, r)}} \in B\left(x, l^{k} r\right)\right) \\
& =1-\mathbb{P}^{x}\left(X_{\tau_{B(x, r)}} \notin B\left(x, l^{k} r\right)\right) \\
& \geq 1-\mathbb{E}^{x}\left[\int_{0}^{\tau_{B(x, r)}} J\left(X_{s}, B\left(x, l^{k} r\right)^{c}\right) d s\right] \\
& \geq 1-c_{1} \frac{\operatorname{Ext}(x, r)}{\operatorname{Ext}\left(x, l^{k} r\right)} \geq 1-c_{2} \rho^{k},
\end{aligned}
$$

where in the third inequality we have used $\mathrm{IJ}_{\mathrm{Ext}, \leq}$ and (5.2), and the last inequality follows from Assumption 1.13(iv). In particular, taking $k \geq 1$ large enough such that $c_{2} \rho^{k} \leq 1 / 2$, we have

$$
\mathbb{P}^{x}\left(\sigma_{B\left(x, l^{k} r\right) \backslash B(x, r)}<\tau_{D}\right) \geq 1 / 2 .
$$

Let $\delta \in(0,1)$ be the constant in EHI. By VD, there exists $L_{*}=L_{*}(l, k) \in \mathbb{N}$ (independent of $x$ and $r$ ) such that $\overline{B\left(x, l^{k} r\right)} \backslash B(x, r) \subset \cup_{i=1}^{L_{*}} B\left(x_{i}, \delta r / 2\right)$ for some $x_{i} \in B\left(x, l^{k} r\right) \backslash B(x, r)$, $i=1, \cdots, L_{*}$, see e.g., [KT, Lemma 3.1]. Let $\nu$ be the relative capacitary measure for $B\left(x, l^{k} r\right) \backslash$ $B(x, r)$ with respect to $G_{D}(x, y)$, which is supported on $\overline{B\left(x, l^{k} r\right)} \backslash B(x, r)$. By applying EHI 
for $G_{D}(x, \cdot)$ on each $B\left(x_{i}, r / 2\right)$, we have

$$
\begin{aligned}
\mathbb{P}^{x}\left(\sigma_{B\left(x, l^{k} r\right) \backslash B(x, r)}<\tau_{D}\right) & =G_{D} \nu(x)=\int_{\overline{B\left(x, l^{k} r\right) \backslash B(x, r)}} G_{D}(x, z) \nu(d z) \\
& \leq \sum_{i=1}^{L_{*}} \int_{B\left(x_{i}, \delta r / 2\right)} G_{D}(x, z) \nu(d z) \\
& \asymp \sum_{i=1}^{L_{*}} \operatorname{essinf}_{y \in B\left(x_{i}, \delta r / 2\right)} G_{D}(x, y) \nu\left(B\left(x_{i}, \delta r / 2\right)\right) \\
& \leq \operatorname{Cap}\left(B\left(x, l^{k} r\right), D\right) \sum_{i=1}^{L_{*}} \operatorname{essinf}_{y \in B\left(x_{i}, \delta r / 2\right)} G_{D}(x, y) \\
& \leq c_{3} \operatorname{Cap}(B(x, r), B(x, 2 r)) \sum_{i=1}^{L_{*}} \operatorname{essinf}_{y \in B\left(x_{i}, \delta r / 2\right)} G_{D}(x, y),
\end{aligned}
$$

where in the last inequality (with $c_{3}=c_{3}(l, k)>0$ ) is due to (1.10). Inequalities (5.5) and (5.6) imply that

$$
\sum_{i=1}^{L_{*}} \operatorname{ess~inf}_{y \in B\left(x_{i}, \delta r / 2\right)} G_{D}(x, y) \geq \frac{c_{4}}{\operatorname{Cap}(B(x, r), B(x, 2 r))} .
$$

Noting that $B\left(x_{i}, \delta r / 2\right) \subset B\left(x_{i}, r / 2\right) \subset B\left(x,\left(l^{k}+1 / 2\right) r\right) \backslash B(x, r / 2)$ for $1 \leq i \leq L_{*}$, we have

$$
\begin{aligned}
\int_{B\left(x,\left(l^{k}+1 / 2\right) r\right) \backslash B(x, r / 2)} G_{D}(x, z) \mu(d z) & \geq L_{*}^{-1} \sum_{i=1}^{L_{*}} \int_{B\left(x_{i}, \delta r / 2\right)} G_{D}(x, z) \mu(d z) \\
& \geq L_{*}^{-1} \sum_{i=1}^{L_{*}} \operatorname{ess~inf}_{y \in B\left(x_{i}, \delta r / 2\right)} G_{D}(x, y) \mu\left(B\left(x_{i}, \delta r / 2\right)\right) \\
& \geq c_{5} \operatorname{Ext}(x, r),
\end{aligned}
$$

where in the last inequality we used VD and (5.7). Hence taking $r^{*}=l^{k+1} r$ and using Assumption 1.13(iv), we have

$$
\mathrm{E}^{x}\left[\tau_{B\left(x, r^{*}\right)}\right] \geq \int_{B\left(x, r^{*}\right) \backslash B\left(x, r^{*} /\left(2 l^{k+1}\right)\right)} G_{B\left(x, r^{*}\right)}(x, z) \mu(d z) \geq c_{5} \operatorname{Ext}\left(x, r^{*} / l^{k+1}\right) \geq c_{6} \operatorname{Ext}\left(x, r^{*}\right) .
$$

The proof is complete.

Proof of Theorem 1.16. According to Lemmas 5.2 and 5.3, we know that under Assumptions 1.13 and 1.14, EHI and $\mathrm{IJ}_{\text {Ext, }} \leq$ imply $\mathrm{E}_{\text {Ext }}$. Using Remark 1.5 and (1.9), we find that $\mathrm{J}_{\mathrm{Ext}, \leq}$ implies $\mathrm{IJ}_{\mathrm{Ext}, \leq}$. Note that in the statement of Corollary 1.12, we indeed have that if $\mathrm{J}_{\phi}$ holds (without $\mathrm{E}_{\phi}$ ), then

$$
\mathrm{FK}(\phi)+\mathrm{PI}(\phi)+\operatorname{CSJ}(\phi) \Longrightarrow \operatorname{EHI}(\phi),
$$

which can be seen from Theorem 1.11 (i) and (ii). Therefore, the desired assertion follows from Corollary 1.12 . 


\section{Example: symmetric jump processes of variable orders}

In this section, we apply the main results of this paper to show that $\mathrm{WEHI}^{+}(\phi)$ and $\mathrm{EHI}(\phi)$ hold for a symmetric stable-like process on $\mathbb{R}^{d}$ of variable order with state-dependent scale function $\phi(x, r)$. This example is a modification from $\left[\mathrm{BKK}\right.$, Example 2.3]. Let $\alpha: \mathbb{R}^{d} \rightarrow\left[\alpha_{1}, \alpha_{2}\right] \subset(0,2)$ be such that

$$
|\alpha(x)-\alpha(y)| \leq \frac{c}{\log (2 /|x-y|)} \quad \text { for }|x-y|<1
$$

holds with some constant $c>0$. Suppose that

$$
\frac{c_{1}}{|x-y|^{d+\alpha(x) \wedge \alpha(y)}} \leq J(x, y) \leq \frac{c_{2}}{|x-y|^{d+\alpha(x) \vee \alpha(y)}} \quad \text { for }|x-y| \leq 1
$$

and

$$
J(x, y) \asymp \frac{1}{|x-y|^{d+\alpha_{1}}} \quad \text { for }|x-y|>1 .
$$

Define

$$
\phi(x, r)= \begin{cases}r^{\alpha(x)}, & 0<r \leq 1 \\ r^{\alpha_{1}}, & r>1\end{cases}
$$

We claim that $\phi(x, r)$ has properties (1.5) and (1.6). Note that for every $x \in \mathbb{R}^{d}$ and $0<r \leq R$,

$$
\frac{\phi(x, R)}{\phi(x, r)}= \begin{cases}\left(\frac{R}{r}\right)^{\alpha(x)}, & 0<r \leq R \leq 1 \\ \frac{R^{\alpha_{1}}}{r^{\alpha(x)},}, & 0<r \leq 1<R \\ \left(\frac{R}{r}\right)^{\alpha_{1}}, & 1<r \leq R .\end{cases}
$$

Since $\alpha(x) \in\left[\alpha_{1}, \alpha_{2}\right]$, it is clear that

$$
\left(\frac{R}{r}\right)^{\alpha_{1}} \leq \frac{\phi(x, R)}{\phi(x, r)} \leq\left(\frac{R}{r}\right)^{\alpha_{2}}
$$

and so $\phi(x, r)$ satisfies condition (1.5). Note that $\sup _{0<r \leq 1} \log (1 / r) / \log (2 / r)<\infty$. Thus by assumption (6.1), there is a constant $c_{3} \geq 1$ so that for every $0<r \leq 1$ and for any $x, y \in \mathbb{R}^{d}$ with $|x-y| \leq r, \phi(x, r)=r^{\alpha(x)} \leq c_{3} r^{\alpha(y)}=c_{3} \phi(y, r)$. When $r>1, \phi(x, r)=r^{\alpha_{1}}=\phi(y, r)$ for every $x, y \in \mathbb{R}^{d}$. Hence $\phi(x, r)$ satisfies (1.6).

We next verify that $\mathrm{J}_{\phi}$ holds for $J(x, y)$. Indeed, by (6.1) there is a constant $c_{4} \geq 1$ such that for all $x, y \in \mathbb{R}^{d}$ with $|x-y| \leq 1$,

$$
1 \leq \frac{|x-y|^{\alpha(x) \wedge \alpha(y)}}{|x-y|^{\alpha(x)}} \leq|x-y|^{-|\alpha(x)-\alpha(y)|}=\exp (|\alpha(x)-\alpha(y)| \log (1 /|x-y|)) \leq c_{4} .
$$

Similarly, there is a constant $c_{5}>0$ such that for all $x, y \in \mathbb{R}^{d}$ with $|x-y| \leq 1$,

$$
c_{5} \leq \frac{|x-y|^{\alpha(x) \vee \alpha(y)}}{|x-y|^{\alpha(x)}} \leq 1 .
$$

Therefore by the definition of $\phi(x, r)$, we have

$$
J(x, y) \asymp \frac{1}{|x-y|^{d} \phi(x,|x-y|)}, \quad x, y \in \mathbb{R}^{d} ;
$$


that is, $\mathrm{J}_{\phi}$ holds.

Define a Dirichlet form $(\mathcal{E}, \mathcal{F})$ on $L^{2}\left(\mathbb{R}^{d} ; d x\right)$ as follows:

$$
\mathcal{E}(f, f)=\int_{\mathbb{R}^{d} \times \mathbb{R}^{d} \backslash \operatorname{diag}}(f(y)-f(x))^{2} J(x, y) d x d y
$$

and $\mathcal{F}$ is the closure the class of Lipschitz functions on $\mathbb{R}^{d}$ with compact support with respect to the norm $\mathcal{E}_{1}(f, f)^{1 / 2}:=\left(\mathcal{E}(f, f)+\|f\|_{L^{2}\left(\mathbb{R}^{d} ; d x\right)}^{2}\right)^{1 / 2}$. By BBCK, Theorem 1.3], there exists $\mathcal{N} \subset \mathbb{R}^{d}$ having zero capacity with respect to the Dirichlet form $(\mathcal{E}, \mathcal{F})$, and there is a conservative and symmetric Hunt process $X:=\left(X_{t}, t \geq 0, \mathbb{P}^{x}\right)$ with state space $\mathbb{R}^{d} \backslash \mathcal{N}$. Note that, in the present setting $X$ is a symmetric jump process of variable order. Furthermore, since

$$
J(x, y) \geq \frac{c_{6}}{|x-y|^{d+\alpha_{1}}}, \quad x, y \in \mathbb{R}^{d},
$$

comparing it with rotationally symmetric $\alpha_{1}$-stable process, we have the following Nash inequality:

$$
\|f\|_{2}^{2+2 \alpha_{1} / d} \leq c_{7} \mathcal{E}(f, f)\|f\|_{1}^{2 \alpha_{1} / d}, \quad f \in \mathcal{F} .
$$

Hence, for any $x, y \in \mathbb{R}^{d} \backslash \mathcal{N}$ and $t>0$, the heat kernel $p(t, x, y)$ of the process $X$ exists and satisfies that

$$
p(t, x, y) \leq c_{8} t^{-d / \alpha_{1}} .
$$

By [BKK, Theorem 3.5], we know that under the present setting, $p(t, x, y)$ can be chosen to be jointly continuous in $(x, y)$ for every fixed $t>0$. Therefore, without loss of generality, we can assume the exceptional set $\mathcal{N} \subset \mathbb{R}^{d}$ above to be empty, i.e., $\mathcal{N}=\emptyset$.

Proposition 6.1. Let $X$ be the process defined above. Then the following holds.

(i) For any $x \in \mathbb{R}^{d}$ and $r>0$,

$$
\mathbb{E}^{x}\left[\tau_{B(x, r)}\right] \asymp \phi(x, r) .
$$

(ii) For any $x \in \mathbb{R}^{d}$ and $r>0$,

$$
\operatorname{Cap}(B(x, r / 2), B(x, r)) \asymp r^{d} / \phi(x, r) .
$$

(iii) Both $\mathrm{WEHI}^{+}(\phi)$ and $\mathrm{EHI}(\phi)$ hold for the process $X$.

Proof. (i) It is clear that there is a constant $c_{1}>0$ so that

$$
\int_{\{|x-y| \geq 1\}} J(x, y) d y \leq c_{1} \quad \text { for every } x \in \mathbb{R}^{d} .
$$

Thus, according to [BKK, Example 2.3 and Theorem 2.1], for all $x \in \mathbb{R}^{d}$ and $0<r \leq 1$,

$$
\mathbb{P}^{x}\left(\tau_{B(x, r)} \leq t\right) \leq c_{2} t r^{-\alpha(x)} .
$$

Hence, for all $x \in \mathbb{R}^{d}$ and $0<r \leq 1$,

$$
\mathbb{E}^{x}\left[\tau_{B(x, r)}\right] \geq \frac{1}{2 c_{2}} r^{\alpha(x)} \mathbb{P}^{x}\left(\tau_{B(x, r)} \geq \frac{1}{2 c_{2}} r^{\alpha(x)}\right) \geq \frac{1}{4 c_{2}} r^{\alpha(x)} .
$$


As mentioned just before this proposition, the heat kernel $p(t, x, y)$ of the process $X$ exists such that (6.4) holds for all $x, y \in \mathbb{R}^{d}$ and $t>0$. On the other hand, it immediately follows from the definition of $J(x, y)$ that there is a constant $c_{3}>0$ such that

$$
\sup _{x} \int_{B(x, r)}|x-y|^{2} J(x, y) d y \leq c_{3} r^{2-\alpha_{1}}
$$

and

$$
\sup _{x} \int_{B(x, r)^{c}} J(x, y) d y \leq c_{3} r^{-\alpha_{1}}
$$

hold for all $r>1$. Then, by using the Davies argument and the Meyer decomposition, we can obtain that for all $t>0$ and $x, y \in \mathbb{R}^{d}$ with $|x-y|>1$,

$$
p(t, x, y) \leq \frac{c_{4}}{t^{d / \alpha_{1}}}\left(1+\frac{|x-y|}{t^{1 / \alpha_{1}}}\right)^{-d-\alpha_{1}} .
$$

In fact, this can be verified by following the proof of [BGK, Theorem 1.4] line by line (see [BGK, Subsection 3.2, Page 153-155] for more details) as well as choosing the truncated constant $K>1$ and replacing $\alpha$ and $\beta$ by $d$ and $\alpha_{1}$ respectively. Note that, upper bound estimate (6.6) says that for $x, y \in \mathbb{R}^{d}$ with $|x-y|>1, p(t, x, y)$ is bounded, up to a constant multiple, by that of rotationally symmetric $\alpha_{1}$-stable processes on $\mathbb{R}^{d}$. Furthermore, it is easy to see that this process is conservative (see e.g. [MUW, Theorem 1.1]). Therefore, we can arrive at that for all $x \in \mathbb{R}^{d}$ and $r>1$,

$$
\mathbb{E}^{x}\left[\tau_{B(x, r)}\right] \geq c_{5} r^{\alpha_{1}}
$$

Indeed, by (6.6) and the conservativeness of the process $X$, we know that for all $x \in \mathbb{R}^{d}, t>0$ and $r>1 \vee t^{1 / \alpha_{1}}$,

$$
\mathbb{P}^{x}\left(\left|X_{t}-x\right| \geq r\right)=\int_{B(x, r)^{c}} p(t, x, y) d y \leq \frac{c_{4}}{t^{d / \alpha_{1}}} \int_{B(x, r)^{c}}\left(1+\frac{|x-y|}{t^{1 / \alpha_{1}}}\right)^{-d-\alpha_{1}} d y \leq \frac{c_{6} t}{r^{\alpha_{1}}} .
$$

This along with the strong Markov property of $X$ yields that for all $x \in \mathbb{R}^{d}, t>0$ and $r \geq$ $2\left(1 \vee(2 t)^{1 / \alpha_{1}}\right)$,

$$
\begin{aligned}
\mathbb{P}^{x}\left(\tau_{B(x, r)} \leq t\right) & \leq \mathbb{P}^{x}\left(\tau_{B(x, r)} \leq t,\left|X_{2 t}-x\right| \leq r / 2\right)+\mathbb{P}^{x}\left(\left|X_{2 t}-x\right| \geq r / 2\right) \\
& \leq \sup _{z \in B(x, r)^{c}} \sup _{s \leq t} \mathbb{P}^{z}\left(\left|X_{2 t-s}-z\right| \geq r / 2\right)+\mathbb{P}^{x}\left(\left|X_{2 t}-x\right| \geq r / 2\right) \\
& \leq \frac{c_{7} t}{r^{\alpha_{1}}}
\end{aligned}
$$

Hence, there are constants $r_{0} \geq 1$ and $c_{8}>0$ such that for all $x \in \mathbb{R}^{d}$ and $r \geq r_{0}$,

$$
\mathbb{P}^{x}\left(\tau_{B(x, r)} \leq c_{8} r^{\alpha_{1}}\right) \leq 1 / 2
$$

In particular, for all $x \in \mathbb{R}^{d}$ and $r \geq r_{0}$,

$$
\mathbb{E}^{x}\left[\tau_{B(x, r)}\right] \geq c_{8} r^{\alpha_{1}} \mathbb{P}^{x}\left(\tau_{B(x, r)} \geq c_{8} r^{\alpha_{1}}\right) \geq c_{8} r^{\alpha_{1}} / 2 .
$$

Note that for all $x \in \mathbb{R}^{d}$ and $1<r \leq r_{0}$, we have by (6.5)

$$
\mathbb{E}^{x}\left[\tau_{B(x, r)}\right] \geq \mathbb{E}^{x}\left[\tau_{B(x, 1)}\right] \geq \frac{1}{4 c_{2}} .
$$


Combining both estimates above, we prove (6.7).

Now we consider upper bound for $\mathbb{E}^{x}\left[\tau_{B(x, r)}\right]$. First, assume $r \leq 1$. Note that the sum $\sum_{s \leq t \wedge \tau_{B(x, r)}} \mathbf{1}_{\left\{\left|X_{s}-X_{s-}\right|>2 r\right\}}$ is 1 if there is a jump of size at least $2 r$ by $t \wedge \tau_{B(x, r)}$, in which case the process exits $B(x, r)$ by time $t$. It is 0 if there is no such jump. So, for all $y \in B(x, r)$,

$$
\begin{aligned}
\mathbb{P}^{y}\left(\tau_{B(x, r)} \leq t\right) & \geq \mathbb{E}^{y}\left[\sum_{s \leq t \wedge \tau_{B(x, r)}} \mathbf{1}_{\left.\left\{\left|X_{s}-X_{s-}\right|>2 r\right\}\right]} \int_{B\left(X_{s}, 2 r\right)^{c}} J\left(X_{s}, z\right) d z d s\right. \\
& =\mathbb{E}^{y} \int_{0}^{t \wedge \tau_{B(x, r)}} \int \geq \frac{c_{9} t \mathbb{P}^{y}\left(\tau_{B(x, r)}>t\right)}{\phi(x, r)}, \\
& \geq \frac{c_{9} \mathbb{E}^{y}\left(t \wedge \tau_{B(x, r)}\right)}{\phi(x, r)} \geq
\end{aligned}
$$

where in the second inequality we have used the facts that

$$
\phi(x, r) \asymp \phi(y, r), \quad|x-y| \leq r
$$

and

$$
\int_{B(x, 2 r)^{c}} J(x, z) d z \geq c_{10}\left(\int_{B(x, 2) \backslash B(x, 2 r)} \frac{1}{|x-z|^{d+\alpha(x)}} d z+\int_{B(x, 2)^{c}} \frac{1}{|x-z|^{d+\alpha_{1}}} d z\right) \asymp r^{-\alpha(x)} .
$$

Therefore,

$$
\mathbb{P}^{y}\left(\tau_{B(x, r)}>t\right) \leq 1-\frac{c_{9} t \mathbb{P}^{y}\left(\tau_{B(x, r)}>t\right)}{\phi(x, r)} .
$$

Taking $t=c_{9}^{-1} \phi(x, r)$ so that $\frac{c_{9} t}{\phi(x, r)}=1$, we obtain that for all $y \in B(x, r)$,

$$
\mathbb{P}^{y}\left(\tau_{B(x, r)}>t\right) \leq \frac{1}{2}
$$

Using the strong Markov property at time $m t$ for $m=1,2, \ldots$,

$$
\mathbb{P}^{x}\left(\tau_{B(x, r)}>(m+1) t\right) \leq \mathbb{E}^{x}\left(\mathbb{P}^{X_{m t}}\left(\tau_{B(x, r)}>t\right) ; \tau_{B(x, r)}>m t\right) \leq \frac{1}{2} \mathbb{P}^{x}\left(\tau_{B(x, r)}>m t\right) .
$$

By induction $\mathbb{P}^{x}\left(\tau_{B(x, r)}>m t\right) \leq 2^{-m}$. With this choice of $t$, we have that for all $x \in \mathbb{R}^{d}$ and $r \in(0,1]$

$$
\mathbb{E}^{x}\left[\tau_{B(x, r)}\right] \leq c_{11} r^{\alpha(x)}
$$

It is easily seen from (6.4) that for all $x, x_{0} \in \mathbb{R}^{d}$ and $t>0$,

$$
\mathbb{P}^{x}\left(X_{t} \in B\left(x_{0}, r\right)\right) \leq c_{12} r^{d} t^{-d / \alpha_{1}} .
$$

For $r \geq 1$, taking $t=\left(2 c_{12}\right)^{\alpha_{1} / d} r^{\alpha_{1}}$ so that $c_{12} r^{d} t^{-d / \alpha_{1}}=1 / 2$, we find that

$$
\mathbb{P}^{x}\left(\tau_{B\left(x_{0}, r\right)}>t\right) \leq \mathbb{P}^{x}\left(X_{t} \in B\left(x_{0}, r\right)\right) \leq \frac{1}{2}
$$

Using the strong Markov property of $X$ again, we arrive at that for all $x, x_{0} \in \mathbb{R}^{d}, \mathbb{P}^{x}\left(\tau_{B\left(x_{0}, r\right)}>\right.$ $k t) \leq 2^{-k}$ and so for all $x \in \mathbb{R}^{d}$ and $r>1$,

$$
\mathbb{E}^{x}\left[\tau_{B(x, r)}\right] \leq c_{13} r^{\alpha_{1}} .
$$


(ii) This follows immediately from Proposition 5.1 and the assertion (i).

(iii) EHR holds by [BKK, Theorem 3.1]. On the other hand, as we noted in (6.3), $\mathrm{J}_{\phi}$ holds, while $\mathrm{E}_{\phi}$ is established in (i). Thus according to Propositions 4.5 4.7, we have $\operatorname{FK}(\phi), \operatorname{PI}(\phi)$ and $\operatorname{CSJ}(\phi)$ for this symmetric non-local Dirichlet form. The desired conclusion now follows from Corollary 1.12 ,

Acknowledgement. We thank the referee for helpful comments on the paper.

\section{References}

[BBCK] M. T. Barlow, R. F. Bass, Z.-Q. Chen and M. Kassmann. Non-local Dirichlet forms and symmetric jump processes. Trans. Amer. Math. Soc. 361 (2009), 1963-1999.

[BGK] M. T. Barlow, A. Grigor'yan and T. Kumagai. Heat kernel upper bounds for jump processes and the first exit time. J. Reine Angew. Math. 626 (2009), 135-157.

[BM] M.T. Barlow and M. Murugan. Stability of elliptic Harnack inequality. Preprint 2016, available at arXiv:1610.01255v2.

[B] R. F. Bass. A stability theorem for elliptic Harnack inequalities. J. Europ. Math. Soc. 17 (2013), 856-876.

[BL] R. F. Bass and D. Levin. Harnack inequalities for jump processes. Potential Anal. 17 (2002), 375-388.

[BKK] R. F. Bass, M. Kassmann and T. Kumagai. Symmetric jump processes: localization, heat kernels and convergence. Ann. Inst. Henri Poincaré Probab. Statist. 46 (2010), 59-71.

[BS] K. Bogdan and P. Sztonyk. Harnack's inequality for stable Lévy processes. Potential Anal. 22 (2005), 133-150.

[CS] L. Caffarelli and L. Silvestre. Regularity theory for fully nonlinear integro-differential equations. Comm. Pure Appl. Math. 62 (2009), 597-638.

[CKP1] A.D. Castro, T. Kuusi and G. Palatucci. Local behavior of fractional p-minimizers. Ann. Inst. H. Poincaré Anal. Non Linéaire 33 (2016), 1279-1299.

[CKP2] A.D. Castro, T. Kuusi and G. Palatucci. Nonlocal Harnack inequalities. J. Funct. Anal. 267 (2014), 1807-1836.

[C] Z.-Q. Chen. On notions of harmonicity. Proc. Amer. Math. Soc. 137 (2009), 3497-3510.

[CF] Z.-Q. Chen and M. Fukushima. Symmetric Markov Processes, Time Change, and Boundary Theory. Princeton Univ. Press, 2012.

[CK1] Z.-Q. Chen and T. Kumagai. Heat kernel estimates for stable-like processes on $d$-sets. Stoch. Proc. Their Appl. 108 (2003), 27-62.

[CK2] Z.-Q. Chen and T. Kumagai. Heat kernel estimates for jump processes of mixed types on metric measure spaces. Probab. Theory Relat. Fields 140 (2008), 277-317. 
[CKW1] Z.-Q. Chen, T. Kumagai and J. Wang. Stability of heat kernel estimates for symmetric non-local Dirichlet forms. Preprint 2016, available at arXiv:1604.04035.

[CKW2] Z.-Q. Chen, T. Kumagai and J. Wang. Stability of parabolic Harnack inequalities for symmetric non-local Dirichlet forms. Preprint 2016, available at arXiv:1609.07594.

[ChK] Z.-Q. Chen and K. Kuwae. On subhamonicity for symmetric Markov processes. J. Math. Soc. Japan 64 (2012), 1181-1209.

[ChZ] Z.-Q. Chen and X. Zhang. Hölder estimates for nonlocal-diffusion equations with drifts. Commun. Math. Stat. 2 (2014), 331-348.

[DT] E. Di Benedetto and N. Trudinger. Harnack inequalities for quasi-minima of variational integrals. Ann. Inst. H. Poincaré Anal. Non Linéaire 1 (1984), 295-308.

[DK] B. Dyda and M. Kassmann. Regularity estimates for ellptic nonlocal operators. Preprint 2015, available at arXiv:1509.08320v2.

[FOT] M. Fukushima, Y. Oshima and M. Takeda. Dirichlet Forms and Symmetric Markov Processes. de Gruyter, Berlin, 2nd rev. and ext. ed., 2011.

[GG] M. Giaquinta, and E. Giusti. On the regularity of the minima of variational integrals. Acta Math. 148 (1982), 31-46.

[Giu] E. Giusti. Direct Methods in the Calculus of Variations. World Scientific Publishing Co. Inc., River Edge 2003.

[GH1] A. Grigor'yan and J. Hu. Upper bounds of heat kernels on doubling spaces. Mosco Math. J. 14 (2014), 505-563.

[GH2] A. Grigor'yan and J. Hu. Heat kernels and green functions on metric measure spaces. Canad. J. Math. 66 (2014), 641-699.

[GHH] A. Grigor'yan, E. Hu and J. Hu. Two-sided estimates of heat kernels of jump type Dirichlet forms. Preprint 2016.

[GT] A. Grigor'yan and A. Telcs. Two-sided estimates of heat kernels on metric measure spaces. Ann. Probab. 40 (2012), 1212-1284.

[Ha] W. Hansen. Intrinsic Hölder continuity of harmonic functions. Potential Anal. 47 (2017), $1-12$.

[HN] W. Hansen and I. Netuka. Scaling invariant Harnack inequalities in a general setting. J. Math. Anal. Appl. 444 (2016), 980-999.

[He] J. Heinonen. Lectures on Analysis on Metric Spaces. Springer-Verlag, New York 2001.

[K1] M. Kassmann. Analysis of symmetric Markov jump processes: A localization technique for non-local operators. Habilitationsschrift, University of Bonn 2007.

[K2] M. Kassmann. A priori estimates for integro-differential operators with measurable kernels. Calc. Var. Partial Differ. Equ. 34 (2009), 1-21. 
[KS] J. Kinnunen and N. Shanmugalingam. Regularity of quasi-minimizers on metric spaces. Manuscripta Math. 105 (2001), 401-423.

[Ko] T. Komatsu. Uniform estimates for fundamental solutions associated with non-local Dirichlet forms. Osaka J. Math. 32 (1995), 833-860.

[KT] T. Kumagai and K. Sturm. Construction of diffusion processes on fractals, $d$-sets, and general metric measure spaces. J. Math. Kyoto Univ. 45 (2005), 307-327.

[MUW] J. Masamune, T. Uemura and J. Wang. On the conservativeness and the recurrence of symmetric jump-diffusions. J. Funct. Anal. 26 (2012), 3984-4008.

[MK] A. Mimica and M. Kassmann. Intrinsic scaling properties for nonlocal operators. J. Eur. Math. Soc. 19 (2017), 983-1011.

[M] J. Moser. On Harnack's theorem for elliptic differential equations. Comm. Pure Appl. Math. 14 (1961), 577-591.

[SU] R. Schilling and T. Uemura. On the Feller property of Dirichlet forms generated by pseudo differential operators. Tohoku Math. J. 59 (2007), 401-422.

[Sil] L. Silvestre. Hölder estimates for solutions of integro-differential equations like the fractional Laplace. Indiana Univ. Math. J. 55 (2006), 1155-1174.

[SV] R. Song and Z. Vondraček. Harnack inequality for some classes of Markov processes. Math. Z. 246 (2004), 177-202.

\section{Zhen-Qing Chen}

Department of Mathematics, University of Washington, Seattle, WA 98195, USA

E-mail: zqchen@uw.edu

\section{Takashi Kumagai}

Research Institute for Mathematical Sciences, Kyoto University, Kyoto 606-8502, Japan

Email: kumagai@kurims.kyoto-u.ac.jp

\section{Jian Wang}

College of Mathematics and Informatics \& Fujian Key Laboratory of Mathematical Analysis and Applications (FJKLMAA), 350007, Fuzhou, P.R. China.

Email: jianwang@fjnu.edu.cn 